SFB

Financial risk measures for a

823 network of individual agents holding portfolios of lighttailed objects

0
0
0
$\infty$

Claudia Klüppelberg, Miriam Isabel Seifert

Nr. 12/2019
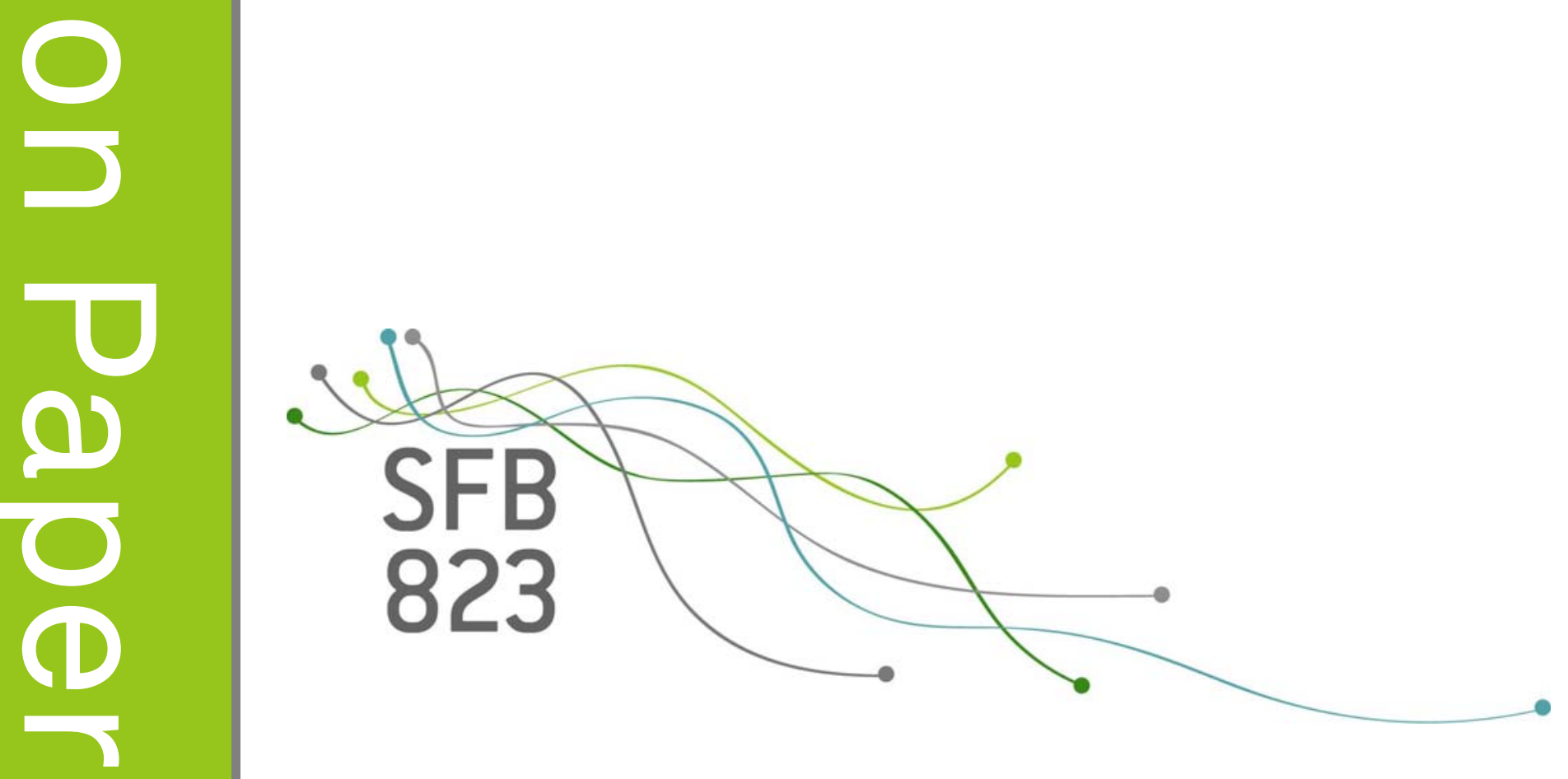



\title{
Financial risk measures for a network of individual agents holding portfolios of light-tailed objects
}

\author{
Claudia Klüppelberg $^{1}$ and Miriam Isabel Seifert ${ }^{2}$
}

May 30, 2019

\begin{abstract}
We investigate a financial network of agents holding portfolios of independent light-tailed risky objects whose losses are asymptotically exponentially distributed with distinct tail parameters. We show that the asymptotic distributions of portfolio losses belong to the class of functional exponential mixtures which we introduce in this paper. We also provide statements for Value-at-Risk and Expected Shortfall risk measures as well as for their conditional counterparts. Compared to heavy tail settings we establish important qualitative differences in the asymptotic behavior of portfolio risks under a light tail assumption which have to be accounted for in practical risk management.
\end{abstract}

Keywords: Asymptotic exponential distribution, Expected Shortfall, Financial network, Risk management, Value-at-Risk

MSC(2010): 60G70, 62E20, 90B10, 91B30

\footnotetext{
${ }^{1}$ Technische Universität München, Boltzmannstraße 3, 85748 Garching, Germany. Email: cklu@tum.de

${ }^{2}$ Ruhr-Universität Bochum, Universitätsstraße 150, 44801 Bochum, Germany. Email: miriam.seifert@rub.de
} 


\section{Introduction}

Studying a network of agents which share financial risks by holding portfolios with different objects subject to random losses is of high relevance for both risk management and financial regulation. By monitoring a financial system, regulators or risk managers should assess risk exposures of different companies or business lines in order to determine capital reserves required in case of unexpectedly large losses. A regulator's or risk manager's assessment requires the following information: What are risk exposures of individual agents? Which are the dominant objects able to cause serious losses to agents or even to the entire system? How does the network structure affect the relationship between individual agent risks and the system risk?

We focus on a system where individual agents, e.g. insurance companies or investment funds, form a financial network by holding portfolios of risky objects. A possible structure of such a network is illustrated by a bipartite graph of agentobject relationships in Figure 1.1. As holding risky portfolios may lead to extreme losses, it is of importance to quantify the tail behavior of the portfolio losses in this network context. Such results are of particular interest for risk managers and regulating authorities, who should facilitate financial stability by monitoring both system and agents' losses. Moreover, they are required for computing the commonly applied risk measures, such as Value-at-Risk, Expected Shortfall, as well as Conditional Value-at-Risk and Expected Shortfall, cf. McNeil et al. [31], Adrian and Brunnermeier [1].

The effects of risk aggregation and risk sharing have been intensively studied in the current literature primarily for heavy-tailed risks with a power tail decay, see Embrechts et al. [13, 14], Kley et al. [23, 24], Lin et al. [26], Ly Vath et al. [27], Mainik and Rüschendorf [28], and Xia [33], among others. In many important settings, however, light-tailed distributions provide a suitable description of risks faced by insurance companies or financial institutions; such tail behavior of risks is typically encountered by household insurances or pension funds holding portfolios which are rebalanced monthly or even quarterly. In particular, the family of generalized hyperbolic distributions with exponential tails is frequently used in the mathematical finance literature (cf. Barndorff-Nielsen et al. [4], McNeil et al. [31]). Further studies dealing with light-tailed distributions are (among others) Asmussen and Albrecher [3], Behme et al. [5], Hernández and Junca [18], Kaas et al. [22], Kyprianou [25] in

the insurance context, and Andersen et al. [2], Cont [9], Cont and Tankov [10] in the financial context.

Up to now, however, there are only few results on risk assessment for portfolios of objects with losses following light-tailed distributions. Jiang and Tang [21] study 


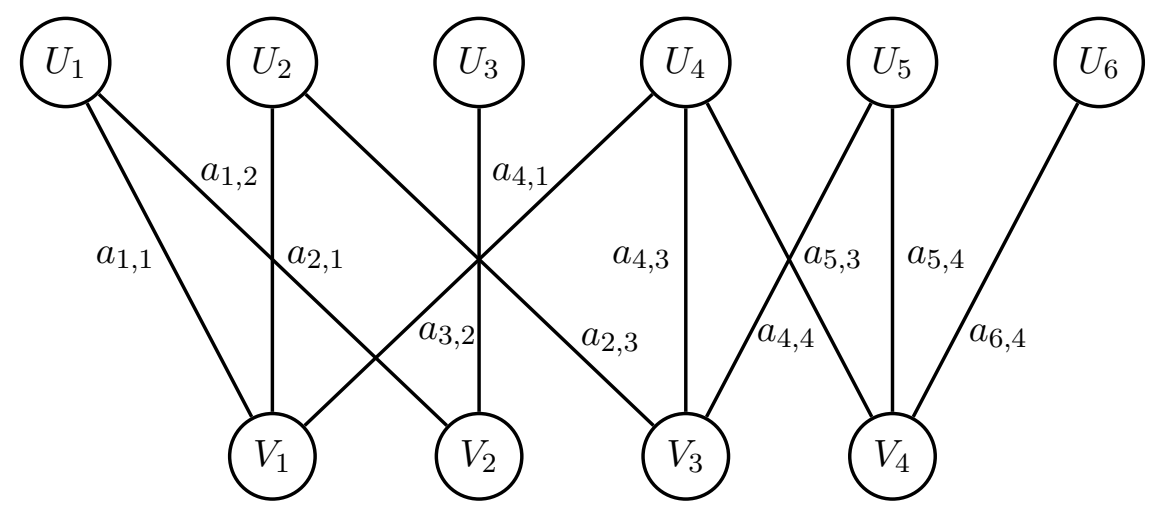

Figure 1.1: Bipartite graph for a network of 4 risky objects causing losses $V_{j}$ held by 6 agents with risk exposures $U_{i}$. The portfolio weights are $a_{i, j}$ for agents $i \in\{1, \ldots, 6\}$ and objects $j \in\{1, \ldots, 4\}$.

the asymptotic behavior of losses for independently and identically exponentially distributed claims. Since they consider all claims with the same parameter of an exponential distribution, the aggregated claim (system loss) follows an Erlang distribution. Mitra and Resnick [32] analyze the sum of two losses with tail-equivalent distributions in the Gumbel max-domain of attraction, which contains distributions with exponential, Gaussian, and log-normal tails as special cases. They assume a certain bivariate dependence structure which leads to the asymptotic independence. Farkas and Hashorva [15] consider portfolios of asymptotically Gaussian losses, they derive limit results for the distribution of portfolio losses and calculate the weak tail dependence coefficient (cf. Coles et al. [8]) for a pair of such portfolios. Dębicki et al. [12] investigate the distribution of losses in the Gumbel max-domain of attraction which are scaled by random factors. However, none of these papers studies consequences of risk sharing in a network or system context.

In this paper we contribute to the current literature by exploring risk aggregation and risk sharing issues for portfolios of light-tailed losses in financial networks. Making assumptions about the full distribution of losses, as e.g. presuming Gaussian or exponential distributions, allows to gain many statements in explicit form. However, it is of high concern to generalize the analysis for light tail settings by making assumptions only about the asymptotic behavior of losses. For this purpose we consider independent light-tailed object losses and only assume that they are asymptotically exponentially distributed. Hence, our framework appears to be rather general and flexible because we impose no restrictions on the finite behavior of losses. Moreover, we investigate the network with objects referring to different risk classes which is formalized by setting distinct tail parameters for all object losses. This is a more general setting than those with the commonly made assumption on tail-equivalent risks for either light- or heavy-tailed losses as e.g. in Jiang and Tang 
[21], Kley et al. [23, 24], Mitra and Resnick [32]. Hence, our study covers a broad family of light-tailed distributions in the context of portfolio risk sharing.

In order to quantify both agents' portfolio and system risks it is necessary to get statements concerning convolutions and mixtures of object loss distributions. For our general setting of asymptotically exponential distributions we obtain them in the form of functional exponential mixtures, where the mixing proportions are not constants but positive asymptotically converging functions. Thus, we extend and generalize the findings for convolutions and mixtures of exponentially distributed random variables, studied among others by Jewell [20] and McLachlan [30]. As functional exponential mixtures allow for a novel and favorable representation of convolutions, we believe that our theoretical contribution would also be of interest beyond the financial network regulation context.

We start the presentation by deriving survival functions for the system loss defined as the sum of all object losses, and for losses of individual agents holding portfolios with selected objects in Theorems 3.2 and 3.6. Then we analyze properties of the introduced functional mixture representations for the survival functions in Remark 3.3 and compare them with classical exponential mixtures. The important result for understanding extreme loss situations is presented in Theorem 4.1, where we show that the dominant impact on individual or system risk is determined by a single (distinct) object, and that generically the risk-dominant object for the system does not coincide with those for individual agents. Moreover, we prove for our light tail setting that asymptotic behavior of individual and system risks is influenced not only by asymptotic but also by non-asymptotic behavior of object losses via their moment-generating functions. Next, in Proposition 4.3 we apply our results in order to quantify the individual portfolio and system risks by computing popular quantilebased risk measures such as Value-at-Risk and Expected Shortfall. We also evaluate conditional risk measures for the network by deducing statements on the Conditional Value-at-Risks and the Conditional Expected Shortfalls in Theorems 5.3 and 5.5. Finally, we compare our findings for systems with light-tailed object losses to the results of Kley et al. [23, 24] for systems with heavy-tailed ones. We point out the substantial qualitative differences in the stochastic behavior for these two settings and provide explanations for them. These differences underscore the importance of our analysis for a proper risk assessment in practical risk management and financial regulation.

Our paper is organized as follows. We first introduce the framework of the study, notation and assumptions in Section 2. In Section 3 we derive the distributions of individual agent risks as well as of the system risk for object losses which follow asymptotically exponential distributions. For this purpose we develop a novel 
concept of functional exponential mixtures which appears to be very useful in our analysis. In Section 4 we exploit our theoretical findings in order to analyze extreme loss situations and to present expressions on marginal quantile-based risk measures. In Section 5 we quantify the interdependence of individual and system risks within the network by deducing results on conditional risk measures. In Section 6 we summarize our findings for portfolios of light-tailed losses and compare them with those established for heavy-tailed ones. The proofs are summarized in Section 7.

\section{Model framework: notation and assumptions}

To formalize the framework for our investigation we introduce a system which consists of $d$ objects and $n$ agents for positive integers $d$ and $n$. Object $j \in \underline{d}:=\{1, \ldots, d\}$ causes a random loss of size $V_{j}>0$ which is shared among the agents holding this object, such that the risk exposure of agent $i$ is given as

$$
U_{i}=\sum_{j \in \underline{d}} a_{i, j} V_{j}, \quad i \in \underline{n}:=\{1, \ldots, n\}
$$

where $a_{i, j}$ is the proportion of object $j$ held by agent $i$. We denote indices referring to agents by $i \in \underline{n}$ and indices referring to objects by $j, k \in \underline{d}$. The weights $a_{i, j}$ for all $d$ objects and $n$ agents are collected into the matrix $A=\left(a_{i, j}\right)_{i \in \underline{n}, j \in \underline{d}}$ of dimension $n \times d$, which is the weighted adjacency matrix to the bipartite graph shown in Figure 1.1. The column-sums of $A$ have to be less or equal to 1:

$$
0 \leq a_{i, j} \leq 1 \quad \text { for all } i \in \underline{n}, j \in \underline{d} ; \quad \sum_{i \in \underline{n}} a_{i, j} \leq 1 \quad \text { for all } j \in \underline{d} .
$$

The risk for object $j$ is covered in total for the boundary case when $\sum_{i \in \underline{n}} a_{i, j}=1$. The system loss is defined as the sum of all object losses:

$$
S=\sum_{j \in \underline{d}} V_{j}
$$

The notation $f(x) \sim g(x)$ means that functions $f(\cdot)$ and $g(\cdot)$ are asymptotically equivalent, i.e., $f(x) / g(x) \rightarrow 1$ for $x \rightarrow \infty$.

Assumption A: let the object losses $V_{j}, j \in \underline{d}$, be stochastically independent positive random variables which follow asymptotic exponential $(\mathcal{A E})$ distributions, i.e. the random variables $V_{j}$ have positive, continuous cumulative distribution functions $F_{V_{j}}(x), x \geq 0$. Their survival functions $P\left\{V_{j}>x\right\}=1-F_{V_{j}}(x)$ satisfy asymptotically for $x \rightarrow \infty$ :

$$
P\left\{V_{j}>x\right\} \sim K_{V_{j}} \exp \left(-\lambda_{j} x\right)
$$


with pairwise distinct tail parameters $\lambda_{j}>0$, i.e. $\lambda_{j} \neq \lambda_{k}$ for $j \neq k$, and positive factors $K_{V_{j}} \in(0, \infty), j \in \underline{d}$. Without loss of generality, we assume

$$
\lambda_{1}<\lambda_{2}<\cdots<\lambda_{d}
$$

where $\lambda_{1}$ is tail parameter of the most risky object.

Representation (2.3) implies that the light-tailed distribution of each object loss $V_{j}$ with support $(0, \infty)$ is well-defined by the following two quantities: the positive tail parameter $\lambda_{j}$ and the positive, continuous factor function $K_{V_{j}}(\cdot)$ with:

$$
K_{V_{j}}(x):=P\left\{V_{j}>x\right\} \exp \left(\lambda_{j} x\right), \quad x \geq 0 .
$$

Hence, the function $K_{V_{j}}(\cdot)$ expresses the deviation from the exponential distribution, and Eqs. (2.3), (2.5) imply that $K_{V_{j}}(0)=1$ and $\lim _{x \rightarrow \infty} K_{V_{j}}(x)=K_{V_{j}} \in(0, \infty)$. Further we write $V_{j} \in \mathcal{A} \mathcal{E}\left(\lambda_{j}, K_{V_{j}}(\cdot)\right)$.

The class of $\mathcal{A E}$ distributions enables to model the stochastic behavior of lighttailed losses in a flexible way. However, to the best of our knowledge, the class of $\mathcal{A E}$ distributions has not been investigated yet in the risk sharing context. In this paper we close this gap by deducing the results for the system and individual portfolio risks.

Our assumption concerning pairwise distinct tail parameters $\lambda_{j} \neq \lambda_{k}$ for $j \neq k$ is more challenging than those of tail-equivalent losses which is commonly used for analyzing both light-tailed (cf. Jiang and Tang [21], Mitra and Resnick [32]) and heavy-tailed risks (cf. Kley et al. [23, 24]). Considering losses with distinct tail parameters is of substantial importance, as such losses can be seen as caused by objects referring to different risk classes. Up to now, distinct tail parameters have been assumed for the analysis of mixtures of exponential distributions by e.g. Bergel and Egídio dos Reis [6], McLachlan [30]. In this paper we impose distinct tail parameters for studying mixtures of $\mathcal{A E}$ distributions. Moreover, in Remark 4.2 below we explain how to handle those cases where some (or all) tail parameters $\lambda_{j}$ coincide.

\section{Risk of individual agents and of the system}

We investigate the distributions of individual risk exposures $U_{i}$ of agents $i \in \underline{n}$ from (2.1) and of the system loss $S$ from (2.2) in terms of their survival functions $P\left\{U_{i}>x\right\}$ and $P\{S>x\}$ for $x \in(0, \infty)$. We show that they follow functional exponential mixture distributions and analyze their mixing proportion functions. 
We start by studying the distribution of the system loss $S=\sum_{j \in \underline{d}} V_{j}$ which is a convolution of $\mathcal{A E}$ distributions. In the following remark we present the established results on convolutions of exponential distributions.

Remark 3.1. Let the object losses $V_{j}, j \in \underline{d}, d \geq 2$, be exponentially distributed with densities $f_{V_{j}}(x)=\lambda_{j} \exp \left(-\lambda_{j} x\right), x>0$, and tail parameters $\lambda_{j}<\lambda_{k}$ for $j<k$. Then the factor functions from (2.5) are constant $K_{V_{j}}(\cdot) \equiv 1$, so that this is a special case $\mathcal{A E}\left(\lambda_{j}, 1\right)$ in our further analysis. Consequently, the system loss $S$ follows a socalled generalized exponential mixture distribution, whose survival function satisfies

$$
P\{S>x\}=\sum_{j \in \underline{d}} \pi_{j, d}^{*} \exp \left(-\lambda_{j} x\right), \quad x>0,
$$

with the mixing proportions

$$
\pi_{j, d}^{*}:=\prod_{k \in \underline{d} \backslash\{j\}} \frac{\lambda_{k}}{\lambda_{k}-\lambda_{j}} .
$$

The class of generalized exponential mixtures has been investigated - although not in a system risk context - by e.g. Jewell [20] and McLachlan [30]. This distribution class is also known as generalized Erlang, see e.g. Bergel and Egídio dos Reis [6]. The mixing proportions $\pi_{j, d}^{*}, j \in \underline{d}$, from (3.2) satisfy $\sum_{j \in \underline{d}} \pi_{j, d}^{*}=1$ and alternate in sign with $\pi_{j, d}^{*}$ positive for odd $j$ and negative for even $j$.

Now we consider convolutions of $\mathcal{A E}$ distributions and show in the following theorem that the system loss $S$ for $\mathcal{A E}$ object losses follows a functional mixture of exponential distributions where the mixing proportions are no longer constants $\pi_{j, d}$ but functions $\pi_{j, d}(\cdot)$. Moreover, it holds that the sum $\sum_{j \in \underline{d}} \pi_{j, d}(x)$ depends on $x>0$ and is a positive value generically different from unity.

Theorem 3.2. Let Assumption A hold for a system of risky objects $V_{j}, j \in \underline{d}$. Then there exist continuous, positive functions $\pi_{j, d}(\cdot):(0, \infty) \rightarrow(0, \infty)$, such that the survival function of the system loss $S=\sum_{j \in \underline{d}} V_{j}$ can be represented as:

$$
P\{S>x\}=\sum_{j \in \underline{d}} \pi_{j, d}(x) \exp \left(-\lambda_{j} x\right), \quad x>0 .
$$

The mixing proportion functions could be chosen recursively for $j \in \underline{k}, k \leq d$ :

$$
\pi_{j, k}(x):= \begin{cases}\int_{0}^{x} \pi_{j, k-1}(x-y) \exp \left(\lambda_{j} y\right) \mathrm{d} F_{V_{k}}(y) & \text { for } j \in \underline{k-1}, \\ K_{V_{k}}(x) & \text { for } j=k,\end{cases}
$$

where $K_{V_{k}}(\cdot)$ is defined as in Eq. (2.5). The mixing proportion functions $\pi_{j, d}(\cdot)$ are positive and bounded from above. 
Note that the ordering (2.4) of the tail parameters is important for building the recursion (3.4). Next, we interpret the result of Theorem 3.2 in the following remark and illustrate it in Figure 3.1.

Remark 3.3. (i) The mixing proportion functions $\pi_{j, d}(\cdot), j \in \underline{d}$, in representation (3.3) for the convolution of $V_{j}$ are not uniquely defined, not even up to asymptotic equivalence. Thus, the choice in (3.4) leads - even in the special case of exponentially distributed losses - to non-constant functions $\pi_{j, d}(\cdot)$ in contrast to the constant mixing proportions $\pi_{j, d}^{*}$ presented in (3.2); in general they differ in their sums because $\sum_{j \in \underline{d}} \pi_{j, d}^{*}=1 \neq \sum_{j \in \underline{d}} \pi_{j, d}(x)$, and even asymptotically for $x \rightarrow \infty$ as $\pi_{j, d}(x) \nrightarrow \rightarrow \pi_{j, d}^{*}$ for $j>1$, cf. Theorem 4.1 below. Moreover, our choice in (3.4) guarantees that all mixing proportion functions are strictly positive. Hence, Theorem 3.2 shows that a generalized exponential mixture with sign alternating mixing proportions can be written as a functional exponential mixture with positive mixing proportions.

The functional mixture $P\{S>x\}=\sum_{j \in \underline{d}} \pi_{j, d}^{*}(x) \exp \left(-\lambda_{j} x\right), x>0$, which coincides for exponentially distributed losses with the generalized exponential mixture representation (3.1) with constant sign-alternating mixing proportions, is given for $k \in \underline{d} \backslash\{1\}$ by the recursion:

$$
\pi_{j, k}^{*}(x):= \begin{cases}\int_{0}^{x} \pi_{j, k-1}^{*}(u) \frac{\exp \left(-\lambda_{j} u\right)}{\exp \left(\left(\lambda_{k}-\lambda_{j}\right) x\right)-1} F_{V_{k}}(x-\mathrm{d} u) & \text { for } j \in \underline{k-1}, \\ K_{V_{k}}(x)-\sum_{l \in \underline{k-1}} \pi_{l, k}^{*}(x) & \text { for } j=k,\end{cases}
$$

with the initial value $\pi_{1,1}^{*}(x)=K_{V_{1}}(x)$. In contrast to $\pi_{j, d}(\cdot)$ from (3.4), the recursion in (3.5) provides the product representation in $(3.2)$ with constants $\pi_{j, d}^{*}(\cdot) \equiv \pi_{j, d}^{*}$ in the special case of exponentially distributed losses.

(ii) In order to illustrate functional mixtures, we contrast representations:

(A) $P\{S>x\}=\sum_{j \in \underline{d}} \pi_{j, d}^{*} \exp \left(-\lambda_{j} x\right), \quad x>0$,

(B) $P\{S>x\}=\sum_{j \in \underline{d}} \pi_{j, d}(x) \exp \left(-\lambda_{j} x\right), \quad x>0$,

with the constant, real-valued $\pi_{j, d}^{*}$ from (3.2) in (A) and positive functions $\pi_{j, d}(\cdot)$ from (3.4) in (B). E.g., consider a system of three objects with exponentially distributed losses $V_{j}$ with tail parameters $\lambda_{1}=1, \lambda_{2}=2, \lambda_{3}=3$. The survival function $P\{S>x\}$ is given by the aggregation of three mixing components $\pi_{j, d}^{*} \exp \left(-\lambda_{j} x\right)$ for $(\mathrm{A})$ or $\pi_{j, d}(x) \exp \left(-\lambda_{j} x\right)$ for $(\mathrm{B})$, with $j=1,2,3$.

We plot the mixing components and the survival functions in Figure 3.1 all premultiplied with the term $\exp \left(\lambda_{1} x\right)$ for a better visual presentation. For representation (A), left, and (B), right, we display the function $x \mapsto P\{S>x\} \exp \left(\lambda_{1} x\right)$ 


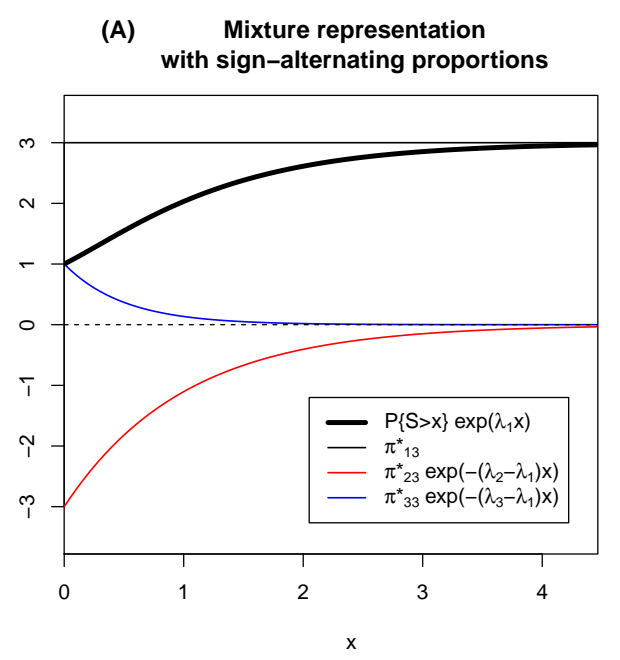

(B) Functional mixture representation with positive proportions

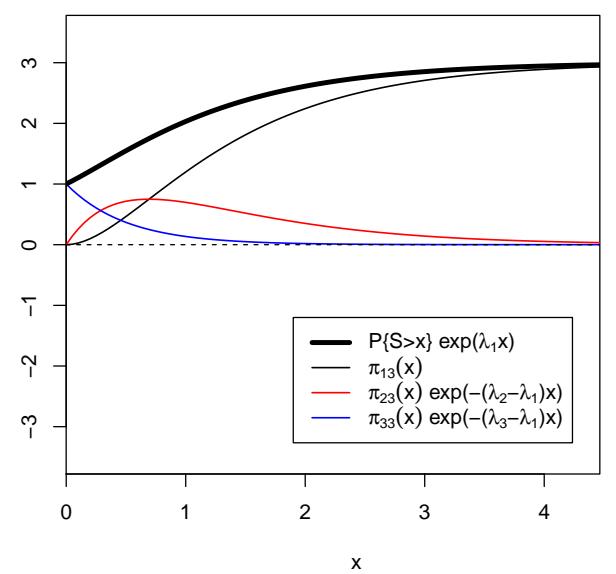

Figure 3.1: Exponential mixture representation in (A), left, versus functional mixture representation from Theorem 3.2 in (B), right, for the system risk $P\{S>x\}$; for more details see Remark 3.3(ii).

in bold solid line which illustrates the behavior of the system risk, as well as the functions $x \mapsto \pi_{j, d}^{*} \exp \left(-\left(\lambda_{j}-\lambda_{1}\right) x\right)$ and $x \mapsto \pi_{j, d}(x) \exp \left(-\left(\lambda_{j}-\lambda_{1}\right) x\right)$ in thin solid lines which illustrate the behavior of three mixing components.

We observe a quite different behavior of the mixing components for representations (A) and (B); in particular, in (B) all curves of the mixing components are located between the zero line and the survival function line, which allows us to evaluate the contribution of each component to the system risk. For extreme loss situations we find $\lim _{x \rightarrow \infty} P\{S>x\} \exp \left(\lambda_{1} x\right)=3=\pi_{1,3}^{*}=\lim _{x \rightarrow \infty} \pi_{1,3}(x)$ as it follows from Theorem 4.1 below.

Next we consider the survival function of the individual loss $U_{i}$ for an arbitrary agent $i \in \underline{n}$ holding a portfolio of objects $V_{j}$ with weights $a_{i, j}$, cf. (2.1), under validity of Assumption A. Let $D_{i}$ be the set of indices for the objects selected by agent $i$ and $d_{i}$ the number of those selected objects, i.e.,

$$
D_{i}:=\left\{j \in \underline{d} \mid a_{i, j}>0\right\}, \quad d_{i}:=\left|D_{i}\right| .
$$

Then the risk exposure $U_{i}=\sum_{j \in D_{i}} a_{i, j} V_{j}$ is the sum of the independent random variables $a_{i, j} V_{j}$ which all follow $\mathcal{A} \mathcal{E}\left(\lambda_{j} / a_{i, j}, K_{V_{j}}\left(\cdot / a_{i, j}\right)\right)$ distributions with tail parameter of $\lambda_{j} / a_{i, j}$ and factors $\left.K_{V_{j}}\left(\cdot / a_{i, j}\right)\right)$ for $j \in D_{i}$. We assume that all $d_{i}$ tail parameters $\lambda_{j} / a_{i, j}$ are pairwise distinct and their ordering is denoted as follows:

$$
D_{i}=:\left\{i(m) \mid m \in \underline{d_{i}}\right\} \quad \text { with } \quad \frac{\lambda_{i(1)}}{a_{i, i(1)}}<\frac{\lambda_{i(2)}}{a_{i, i(2)}}<\cdots<\frac{\lambda_{i\left(d_{i}\right)}}{a_{i, i\left(d_{i}\right)}},
$$


such that $i(1)$ denotes the object with the smallest value of $\lambda_{j} / a_{i, j}$ from all objects $j \in D_{i}$ selected by agent $i$. Now we deduce $P\left\{U_{i}>x\right\}$ for the individual losses analogously to the result on the system loss $S$ in Theorem 3.2:

Corollary 3.4. The survival function of the individual loss $U_{i}=\sum_{j \in \underline{d}} a_{i, j} V_{j}$ for agent $i \in \underline{n}$ can be represented as:

$$
P\left\{U_{i}>x\right\}=\sum_{j \in D_{i}} \pi_{i, j, d_{i}}(x) \exp \left(-\lambda_{j} x / a_{i, j}\right), \quad x>0,
$$

where the continuous, positive mixing proportion functions $\pi_{i, j, d_{i}}(\cdot):(0, \infty) \rightarrow(0, \infty)$, $j \in D_{i}$, can be chosen recursively for $m \in \underline{k}, k \leq d_{i}$ as:

$$
\begin{aligned}
& \pi_{i, i(m), k}(x) \\
&:= \begin{cases}\int_{0}^{x} \pi_{i, i(m), k-1}\left(x-a_{i, i(k)} y\right) \exp \left(\frac{a_{i, i(k)} \lambda_{i(m)}}{a_{i, i(m)}} y\right) \mathrm{d} F_{V_{i(k)}}(y) & \text { for } m \in \underline{k-1} \\
K_{V_{i(k)}}\left(x / a_{i, i(k)}\right) & \text { for } m=k .9\end{cases}
\end{aligned}
$$

For the asymptotic analysis of the mixing proportion functions for the system and individual risks we provide the following lemma concerning the object loss distributions.

Lemma 3.5. Let the object loss $V_{k}$ follow $\mathcal{A E}\left(\lambda_{k}, K_{V_{k}}(\cdot)\right)$ distribution according to Assumption $A$. Then the moment-generating function $\phi_{V_{k}}(t):=E\left[\exp \left(t V_{k}\right)\right]$ of $V_{k}$ exists, is finite for all $t<\lambda_{k}$ with singularity at $\lambda_{k}$.

The mixing proportion functions for the distributions of individual and system loss converge to positive finite limits, as we prove it in the following theorem.

Theorem 3.6. Let Assumption A hold for a system of risky objects $V_{j}, j \in \underline{d}$, and let agent $i \in \underline{n}$ hold a portfolio with weights according to (3.7). Then the mixing proportion functions $\pi_{j, d}(\cdot)$ from (3.4) and $\pi_{i, j, d_{i}}(\cdot)$ from (3.9) have finite and strictly positive limits for $x \rightarrow \infty$. These limits are given explicitly as:

$$
\begin{aligned}
\pi_{j, d} & :=\lim _{x \rightarrow \infty} \pi_{j, d}(x)=K_{V_{j}} \prod_{k=j+1}^{d} \phi_{V_{k}}\left(\lambda_{j}\right), \\
\pi_{i, i(m), d_{i}} & :=\lim _{x \rightarrow \infty} \pi_{i, i(m), d_{i}}(x)=K_{V_{i(m)}} \prod_{k=m+1}^{d_{i}} \phi_{V_{i(k)}}\left(\frac{a_{i, i(k)} \lambda_{i(m)}}{a_{i, i(m)}}\right),
\end{aligned}
$$

with $j \in D_{i}, m \in \underline{d_{i}}$, and the moment-generating function $\phi_{V_{k}}(\cdot)$ of object loss $V_{k}$ :

$$
\phi_{V_{k}}\left(\lambda_{j}\right):=E\left[\exp \left(\lambda_{j} V_{k}\right)\right]=\int_{0}^{\infty} \exp \left(\lambda_{j} y\right) \mathrm{d} F_{V_{k}}(y) \in(1, \infty), j \in \underline{k-1} .
$$


For $j=d$ or $m=d_{i}$ the empty product in (3.10) or in (3.11) is set equal to 1 according to the common convention.

Theorem 3.6 states that the survival functions of system and individual losses are asymptotically equivalent to non-functional exponential mixtures with positive mixing proportions: it holds that $P\{S>x\} \sim \sum_{j \in d} \pi_{j, d} \exp \left(-\lambda_{j} x\right)$ as well as $P\left\{U_{i}>x\right\} \sim \sum_{j \in D_{i}} \pi_{i, j, d_{i}} \exp \left(-\lambda_{j} x / a_{i, j}\right)$ for $x \rightarrow \infty$. We show that the limit values $\pi_{j, d}$ and $\pi_{i, j, d_{i}}$ depend essentially on the moment-generating functions $\phi_{V_{k}}(\cdot)$ of the object losses. The result in Lemma 3.5 guarantees that the values $\phi_{V_{k}}\left(\lambda_{j}\right)$ for

$j<k$ and $\phi_{V_{i(k)}}\left(a_{i, i(k)} \lambda_{i(m)} / a_{i, i(m)}\right)$ for $m<k$ from the result in Theorem 3.6 are all finite and larger than one, see also (3.7).

For the special case of exponentially distributed losses, the result in Theorem 3.6 shows that mixing proportion functions $\pi_{j, d}(\cdot)$ for $P\{S>x\}$ differ even asymptotically from generalized exponential mixing proportions $\pi_{j, d}^{*}$ given in (3.2) for $j>1$.

\section{Extreme losses in financial network and marginal risk measures}

As particularly extreme losses have the most adverse effects on the stability of financial systems, in this section we derive the asymptotic distributions of portfolio losses and provide statements for the popular quantile-based risk measures such as Value-at-Risk and Expected Shortfall. As we have shown in Theorems 3.2 and 3.6, the distributions for the system loss $S$ and the individual agents' exposures $U_{i}$ can be written as functional exponential mixtures with positive mixing proportion functions $\pi_{j, d}(\cdot)$ and $\pi_{i, j, d_{i}}(\cdot)$ from (3.4) and (3.9), where the limits $\pi_{j, d}=\lim _{x \rightarrow \infty} \pi_{j, d}(x)$ and $\pi_{i, j, d_{i}}=\lim _{x \rightarrow \infty} \pi_{i, j, d_{i}}(x)$ exist, are finite and strictly positive, and, hence, do not influence the asymptotic tail decay.

In the next theorem we state the important result characterizing our financial network with $\mathcal{A} \mathcal{E}$ object losses, namely that both system and individual agent's risk follow - as convolutions of $\mathcal{A E}$ distributions $-\mathcal{A E}$ distributions too. Their asymptotic tail decays are determined by the object with the minimal tail parameter of all object losses $V_{j}$ in the system or of all weighted losses $a_{i, j} V_{j}$ in agent's portfolio:

$$
\lambda_{1}=\min _{j \in \underline{d}} \lambda_{j} \quad \text { and } \quad \mu_{i}:=\min _{j \in D_{i}}\left(\lambda_{j} / a_{i, j}\right) .
$$

Theorem 4.1. Let Assumption A hold for a system of risky objects $V_{j}, j \in \underline{d}$, and let agent $i \in \underline{n}$ hold a portfolio with weights according to (3.7). Then:

(i) the system loss $S=\sum_{j \in \underline{d}} V_{j}$ follows $\mathcal{A} \mathcal{E}\left(\lambda_{1}, K_{S}(\cdot)\right)$ distribution with the factor 
function $K_{S}(x):=\pi_{1, d}(x), x>0$. Asymptotically for $x \rightarrow \infty$ it holds that:

$$
P\{S>x\} \sim K_{S} \exp \left(-\lambda_{1} x\right),
$$

with $K_{S}:=K_{V_{1}} \prod_{k=2}^{d} \phi_{V_{k}}\left(\lambda_{1}\right)$ and the moment-generating function $\phi_{V_{k}}(\cdot)$ of $V_{k}$;

(ii) the individual loss $U_{i}=\sum_{j \in \underline{d}} a_{i, j} V_{j}$ of agent $i \in \underline{n}$ follows $\mathcal{A} \mathcal{E}\left(\mu_{i}, K_{U_{i}}(\cdot)\right)$ distribution with the factor function $K_{U_{i}}(x):=\pi_{i, i(1), d_{i}}(x), x>0$. Asymptotically for $x \rightarrow \infty$ it holds that:

$$
\begin{gathered}
P\left\{U_{i}>x\right\} \sim K_{U_{i}} \exp \left(-\mu_{i} x\right), \\
\text { where } K_{U_{i}}:=K_{V_{i(1)}} \prod_{k=2}^{d_{i}} \phi_{V_{i(k)}}\left(\frac{a_{i, i(k)} \lambda_{i(1)}}{a_{i, i(1)}}\right) \text { with notation } i(k) \text { from (3.7). }
\end{gathered}
$$

For $d=1$ or $d_{i}=1$ the empty product is set equal to 1 according to the common convention.

Theorem 4.1 proves that the class of $\mathcal{A E}$ distributions is closed under scaling and convolution, in contrast to the class of exponential distributions which does not satisfy this closure property under convolution.

Both system loss $S$ and individual loss $U_{i}$ of agent $i \in \underline{n}$ have asymptotically exponential tails, but with possibly different tail decays. The survival function of the system loss is asymptotically proportional to that of the object loss $V_{1}$ with minimum tail parameter $\lambda_{1}$. The asymptotic dominance of this object for the system risk is illustrated in Figure 3.1 where it is to see that - independently from the chosen mixture representation - the function $P\{S>x\} \exp \left(\lambda_{1} x\right)$ converges to the positive limit $\pi_{1, d}=\lim _{x \rightarrow \infty} \pi_{1, d}(x)$. Analogously, the survival function of the individual loss $U_{i}$ is asymptotically proportional to that of the weighted loss $a_{i, i(1)} V_{i(1)}$ with the agent's minimum tail parameter $\mu_{i}$. If agent $i$ does not select the most risky object $V_{1}$ alone in total; i.e., if $a_{i, 1}<1$ and, hence, $\lambda_{1}<\mu_{i}$, the individual risk is asymptotically of lower order than the system risk:

$$
P\left\{U_{i}>x\right\}=o(P\{S>x\}) \text { for } x \rightarrow \infty \text {. }
$$

In contrast to the tail parameter $\lambda_{1}$ of the system loss or to the tail parameter $\mu_{i}$ of the individual loss, both determined by a single (dominant) object, the factors $K_{S}$ or $K_{U_{i}}$ are influenced by all objects in the system or in the agent's portfolio, respectively. We show that the 'closer' the tail parameters of the other objects are to that of the dominant object the larger is the value of the factor $K_{S}$ or $K_{U_{i}}$.

Furthermore, in situations where the agent modifies his portfolio by adding or removing objects, the tail parameter of the agent's $\mathcal{A E}$ distribution remains unchanged 
as long as his dominant tail parameter $\mu_{i}$ remains the same. However, the asymptotic individual risk changes in terms of the factor $K_{U_{i}}$, when adding or removing objects.

In Theorem 4.1 we prove that asymptotic risks of individual agent portfolios and the system depend on their entire factor functions $K_{V_{j}}(\cdot)$ entering the survival functions $P\left\{V_{j}>x\right\}=K_{V_{j}}(x) \exp \left(-\lambda_{j} x\right)$ of the object losses, and not only on their limit values $\lim _{x \rightarrow \infty} K_{V_{j}}(x)$. This influence is determined by the moment-generating functions $\phi_{V_{k}}(\cdot)$ of object losses $V_{k}$. Our result implies that in a system of independent $\mathcal{A E}$ distributed object losses the asymptotic behavior of the system and individual agent risks is essentially influenced by the non-asymptotic behavior of the object loss distributions. This finding points on a qualitative difference to the established results for systems of heavy-tailed risks, where non-asymptotic behavior of object loss distributions does not affect the asymptotic risks in the system, cf. Kley et al. [23].

In the following remark we comment on consequences of relaxing the restriction on pairwise distinct tail parameters which we made in Assumption A.

Remark 4.2. If the tail parameters $\lambda_{j}$ coincide for different objects $j$, then the distribution of the system loss $S$ is a functional mixture of Erlang distributions such that:

$$
P\{S>x\}=\sum_{k \in H} \pi_{k, h}(x) x^{h_{k}-1} \exp \left(-\lambda_{k} x\right), \quad x>0,
$$

where $H \subseteq \underline{d}$ is a set of object indices satisfying $\left\{\lambda_{j} \mid j \in \underline{d}\right\}=\left\{\lambda_{k} \mid k \in H\right\}$ such that $\lambda_{k}<\lambda_{l}$ for $k<l, k, l \in H$. Moreover, $h:=|H|$ denotes the number of pairwise distinct tail parameters in the system and $h_{k}:=\left|\left\{j \in \underline{d} \mid \lambda_{j}=\lambda_{k}\right\}\right|$ denotes the number of tail parameters equal to $\lambda_{k}$.

The asymptotic results extending Theorem 4.1 lead to Erlang tails, in particular, we obtain for the system loss $S$ the tail $\pi_{1, h} x^{h_{1}-1} \exp \left(-\lambda_{1} x\right)$. Hence, $h_{1}$ is the number of asymptotically dominant objects with the minimum tail parameter $\lambda_{1}$. For the individual loss $U_{i}$ this holds, respectively, with a set $H_{i} \subseteq D_{i}$ defined analogously to the set $H$ above.

Based on our results for the distributions of individual agent and system risks, we next provide statements for the marginal risk measures Value-at-Risk and Expected Shortfall which are of high importance in practice. Such results for light-tailed $\mathcal{A E}$ risks amend the corresponding analysis concerning risk measures for heavy-tailed loss distributions, provided among others by Das and Fasen-Hartmann [11], Ibragimov [19], Kley et al. [24], Mainik and Rüschendorf [28].

For level $\alpha \in(0,1)$ we provide results on the Value-at-Risk for the loss from 
object $j \in \underline{d}$ :

$$
\operatorname{Obj} \operatorname{VaR}_{j}(\alpha):=\inf \left\{v \geq 0 \mid P\left\{V_{j}>v\right\} \leq 1-\alpha\right\},
$$

the individual Value-at-Risk for agent $i \in \underline{n}$ :

$$
\text { Ind } \operatorname{VaR}_{i}(\alpha):=\inf \left\{u \geq 0 \mid P\left\{U_{i}>u\right\} \leq 1-\alpha\right\},
$$

and the system Value-at-Risk:

$$
\text { Sys } \operatorname{VaR}(\alpha):=\inf \{s \geq 0 \mid P\{S>s\} \leq 1-\alpha\} .
$$

Moreover, we prove statements for the corresponding Expected Shortfalls which can be interpreted as the expected losses in extreme situations where the given Valueat-Risk is exceeded. Accordingly, for level $\alpha \in(0,1)$ we investigate the Expected Shortfall for the loss of object $j \in \underline{d}$ :

$$
\operatorname{Obj}_{\operatorname{ES}}(\alpha):=E\left[V_{j} \mid V_{j}>\operatorname{Obj}_{\operatorname{VaR}_{j}}(\alpha)\right],
$$

the individual Expected Shortfall for agent $i \in \underline{n}$ :

$$
\text { Ind } \operatorname{ES}_{i}(\alpha):=E\left[U_{i} \mid U_{i}>\operatorname{Ind} \operatorname{VaR}_{i}(\alpha)\right]
$$

and the system Expected Shortfall:

$$
\operatorname{Sys} \operatorname{ES}(\alpha):=E[S \mid S>\operatorname{Sys} \operatorname{VaR}(\alpha)] .
$$

As we show in the following results, the asymptotic behavior of quantile-based risk measures in a system of $\mathcal{A E}$ losses is determined by the minimal tail parameter among those in the agent's portfolio $\mu_{i}$ or among all objects in the system $\lambda_{1}$ as defined in (4.1).

Proposition 4.3. Let Assumptions A hold for a system of risky objects $V_{j}, j \in \underline{d}$, and let agent $i \in \underline{n}$ hold a portfolio with weights according to (3.7). Then:

(i) for the Value-at-Risks it holds asymptotically as $\alpha \uparrow 1$ that:

$$
\begin{aligned}
\operatorname{Obj}_{\operatorname{VaR}_{j}}(\alpha) & \sim \frac{-\ln (1-\alpha)}{\lambda_{j}}, j \in \underline{d}, \\
\operatorname{Ind} \operatorname{VaR}_{i}(\alpha) & \sim \frac{-\ln (1-\alpha)}{\mu_{i}}, i \in \underline{n}, \\
\operatorname{Sys} \operatorname{VaR}(\alpha) & \sim \frac{-\ln (1-\alpha)}{\lambda_{1}} ;
\end{aligned}
$$

(ii) for the Expected Shortfalls it holds asymptotically that:

$$
\begin{aligned}
\lim _{\alpha \uparrow 1}\left(\operatorname{ObjES}_{j}(\alpha)-\operatorname{Obj}_{\left.\operatorname{VaR}_{j}(\alpha)\right)}\right. & =1 / \lambda_{j}, j \in \underline{d}, \\
\lim _{\alpha \uparrow 1}\left(\operatorname{Ind} \operatorname{ES}_{i}(\alpha)-\operatorname{Ind}_{\left.\operatorname{VaR}_{i}(\alpha)\right)}\right. & 1 / \mu_{i}, \quad i \in \underline{n}, \\
\lim _{\alpha \uparrow 1}(\operatorname{Sys} \operatorname{ES}(\alpha)-\operatorname{Sys} \operatorname{VaR}(\alpha)) & =1 / \lambda_{1} .
\end{aligned}
$$


In the following remark we interpret our results on the asymptotic behavior of the Value-at-Risks in a system of $\mathcal{A E}$ object losses.

Remark 4.4. (i) Proposition 4.3 states that all Value-at-Risks tend to infinity if the level $\alpha$ tends to 1 with the same logarithmic rate, so that they are asymptotically proportional to each other and differ only in their proportionality factors. The latter is in each case the reciprocal of the tail parameter for the respective dominant object, i.e. $1 / \mu_{i}$ for the $i$-th agent's portfolio and $1 / \lambda_{1}$ for the system.

(ii) The asymptotic Value-at-Risks are not influenced by the number of objects in the whole system or in the portfolio of agent $i$. Hence, the individual agent's Valueat-Risk remains asymptotically unchanged if he modifies the portfolio by adding or removing objects as long as the dominant tail parameter $\mu_{i}$ remains unchanged.

(iii) Another remarkable property proven in Proposition 4.3 is that the asymptotic Value-at-Risks depend on marginal distributions $P\left\{V_{j}>x\right\}=K_{V_{j}}(x) \exp \left(-\lambda_{j} x\right)$ of object losses only by the dominant tail parameters; i.e. the asymptotic Value-atRisks are independent of the factor functions $K_{V_{j}}(\cdot)$ and their limits $K_{V_{j}}$ for $x \rightarrow \infty$. This is qualitatively different compared to the Value-at-Risk results for heavy-tailed loss distributions, more details are given in Section 6, part (III).

The results in Proposition 4.3 imply that the Expected Shortfalls for objects, agents, as well as for the system are asymptotically equivalent to the respective Value-at-Risks. Moreover, we specify the asymptotic equivalence by establishing that the distance between Expected Shortfall and Value-at-Risk converges to a finite, non-zero limit which depends on the respective dominant tail parameter.

Corollary 4.5. For the Expected Shortfalls it holds for $\alpha \uparrow 1$ that:

$$
\begin{aligned}
\operatorname{ObjES}_{j}(\alpha) & \sim \operatorname{Obj}_{V^{\prime}}(\alpha) \sim \frac{-\ln (1-\alpha)}{\lambda_{j}}, \\
\operatorname{Ind} \operatorname{ES}_{i}(\alpha) & \sim \operatorname{Ind} \operatorname{VaR}_{i}(\alpha) \sim \frac{-\ln (1-\alpha)}{\mu_{i}}, \\
\operatorname{Sys} E S(\alpha) & \sim \operatorname{Sys} \operatorname{VaR}(\alpha) \sim \frac{-\ln (1-\alpha)}{\lambda_{1}} .
\end{aligned}
$$

Due to Corollary 4.5, all properties described in Remark 4.4 for Value-at-Risks are also valid for the Expected Shortfalls.

\section{Conditional risk measures}

To assess the riskiness of a system we quantify not only the marginal risks of individual agents or of the system, but also their interdependence within the network by considering conditional risk measures. Such statements are of particular relevance for regulators of a financial system which should monitor its stability. 
For a network of agents sharing $\mathcal{A E}$ objects we provide results on the Conditional Value-at-Risks (CoVaR) in Theorem 5.3 as well as on the Conditional Expected Shortfalls (CES) in Theorem 5.5. Note that our asymptotic statements are obtained by conditioning on the event that a loss exceeds a given threshold.

For gaining these statements, we focus on equally weighted portfolios usually used in the relevant literature, see e.g. Brechmann et al. [7], Geluk et al. [16], Ibragimov [19]. Then the agent $i$ selects some objects $j \in D_{i}$ and holds the same proportion of each selected object in his portfolio, i.e.,

$$
a_{i, j}=a_{i} \text { for all } j \in D_{i} \text { and some } 0<a_{i} \leq 1
$$

As a consequence, we can simplify the notation from (4.1) using (3.7) as follows:

$$
\lambda_{1}=\min _{j \in \underline{d}} \lambda_{j} \quad \text { and } \quad \lambda_{i(1)}=\min _{j \in D_{i}} \lambda_{j} .
$$

Next we compute the joint probability of individual loss and system loss.

Proposition 5.1. Let Assumption A hold for a system of risky objects $V_{j}, j \in \underline{d}$, and let agent $i \in \underline{n}$ hold a portfolio with weights according to (5.1). Then it follows:

(i) The joint probability of agent's $i$ exposure $U_{i}$ and the system loss $S$ could be written as functional exponential mixture representations:

$$
P\left(U_{i}>u, S>s\right)= \begin{cases}\sum_{k \in \underline{d}} b_{k, u / a_{i}}(s) \exp \left(-\lambda_{k} s\right) & \text { for } u \leq a_{i} s \\ \sum_{j \in D_{i}} b_{j}\left(u / a_{i}\right) \exp \left(-\lambda_{j} u / a_{i}\right) & \text { for } u>a_{i} s\end{cases}
$$

with bounded functions $b_{k, r}(\cdot):[0, \infty) \rightarrow(-\infty, \infty), k \in \underline{d}, r \in[0, \infty)$, which is given in (7.13), and $b_{j}(\cdot):[0, \infty) \rightarrow(-\infty, \infty), j \in D_{i}$, as in $(7.17)$.

(ii) Asymptotically for $s \rightarrow \infty$ and $u>0$ fixed it holds that:

$$
P\left(U_{i}>u, S>s\right) \sim C_{i}\left(u / a_{i}\right) \exp \left(-\lambda_{1} s\right)
$$

with the coefficient

$$
C_{i}\left(u / a_{i}\right):= \begin{cases}\pi_{1, d} & \text { for } \lambda_{1}=\lambda_{i(1)}, \\ \pi_{1, d}-H_{i}\left(u / a_{i}\right) K_{V_{1}} \prod_{k \in \underline{d} \backslash D_{i}, k \neq 1} \phi_{V_{k}}\left(\lambda_{1}\right) & \text { for } \lambda_{1}<\lambda_{i(1)},\end{cases}
$$

where $\phi_{V_{k}}(\cdot)$ is the moment-generating function of $V_{k}, \pi_{1, d}$ from (3.10), and 


$$
\begin{aligned}
H_{i}\left(u / a_{i}\right):= & \int_{0}^{u / a_{i}} \exp \left(\lambda_{1} z\right) \mathrm{d} F_{\sum_{j \in D_{i}} V_{j}}(z) \\
= & 1-\sum_{j \in D_{i}} \pi_{j, d_{i}}\left(u / a_{i}\right) \exp \left(-\left(\lambda_{j}-\lambda_{1}\right) u / a_{i}\right) \\
& +\lambda_{1} \sum_{j \in D_{i}} \int_{0}^{u / a_{i}} \pi_{j, d_{i}}(z) \exp \left(-\left(\lambda_{j}-\lambda_{1}\right) z\right) \mathrm{d} z,
\end{aligned}
$$

where $\pi_{j, d_{i}}(\cdot)$ is defined analogously to $\pi_{j, d}(\cdot)$ in (3.4) but on the subset $D_{i} \subseteq \underline{d}$.

(iii) Asymptotically for $u \rightarrow \infty$ and $s>0$ fixed it holds that:

$$
P\left(U_{i}>u, S>s\right) \sim \pi_{i(1), d_{i}} \exp \left(-\lambda_{i(1)} u / a_{i}\right),
$$

with $\lambda_{i(1)}$ from (5.2) and $\pi_{i(1), d_{i}}=K_{V_{i(1)}} \prod_{k \in D_{i}, k \neq i(1)} \phi_{V_{k}}\left(\lambda_{i(1)}\right)$.

In part (iii) it holds that $\pi_{i(1), d_{i}}=\pi_{i, i(1), d_{i}}$ as defined in (3.11), where the index $i$ becomes redundant due (5.1). The asymptotic form of the joint probability $P\left(U_{i}>\right.$ $u, S>s$ ) for $u \rightarrow \infty$ in (5.4) is independent of the fixed threshold value $s$, and the asymptotic form for $s \rightarrow \infty$ in (5.3) depends on the threshold value $u / a_{i}$ only if agent $i$ does not hold the risk-dominant object with tail parameter $\lambda_{1}$ in his portfolio.

Remark 5.2. By proving Proposition 5.1 the integrals with respect to the functional mixture distributions in our network of $\mathcal{A E}$ losses are more challenging compared to the case with constant mixing proportions, because in general the functions $H_{j}(x):=\pi_{j}(x) F_{V_{j}}(x)$ do not define a measure due to the violation of monotonicity. Consequently, $\int g(z) \mathrm{d} F_{\sum_{j} V_{j}}(z)=\sum_{j} \int g(z) \mathrm{d} H_{j}(z)$ does not hold for functional mixtures $1-F_{\sum_{j} V_{j}}(z)=\sum_{j} \pi_{j, d}(z) \exp \left(\lambda_{j} z\right)$ with non-constant $\pi_{j, d}(\cdot)$. However, for some measure defining function $G$ it holds with integration by parts that:

$$
\int_{a}^{b} G(z) \mathrm{d} F_{\sum_{j} V_{j}}(z)=\int_{a}^{b} P\left\{\sum_{j} V_{j}>z\right\} \mathrm{d} G(z)-\left.P\left\{\sum_{j} V_{j}>z\right\} G(z)\right|_{a} ^{b},
$$

which is very convenient for the functional mixtures under consideration.

Next, for level $\alpha \in(0,1)$ and bounds $u, s \in(0, \infty)$ we evaluate the Conditional Value-at-Risk for an individual agent $i \in \underline{n}$ in a systemic stress situation:

$$
\text { Ind } \operatorname{CoVaR}_{U_{i} \mid S>s}(\alpha):=\inf \left\{u \geq 0 \mid P\left(U_{i}>u \mid S>s\right) \leq 1-\alpha\right\} \text {, }
$$


and the system CoVaR for a situation where agent $i \in \underline{n}$ is in financial distress:

$$
\text { Sys }_{\operatorname{CoVaR}}^{S \mid U_{i}>u}(\alpha):=\inf \left\{s \geq 0 \mid P\left(S>s \mid U_{i}>u\right) \leq 1-\alpha\right\} .
$$

Usually the bounds are chosen as $u=\operatorname{Ind} \operatorname{VaR}(\beta), s=\operatorname{Sys} \operatorname{VaR}(\beta)$ for $\beta \in(0,1)$.

Additionally, as we are interested in the asymptotic analysis of risks, we modify the CoVaR notion by introducing proportionally increasing thresholds for both stress events $\{S>s\}$ and $\left\{U_{i}>\theta s\right\}$ with the scaling factor $\theta \in\left(0, a_{i}\right)$ :

$$
\begin{aligned}
& \operatorname{Ind} \operatorname{CoVaR}_{U_{i} \mid S}(\alpha, \theta):=\inf \left\{s \geq 0 \mid P\left(U_{i}>\theta s \mid S>s\right) \leq 1-\alpha\right\}, \\
& \operatorname{Sys}_{\mathrm{CoVaR}_{S \mid U_{i}}(\alpha, \theta)}:=\inf \left\{s \geq 0 \mid P\left(S>s \mid U_{i}>\theta s\right) \leq 1-\alpha\right\} .
\end{aligned}
$$

Theorem 5.3. Let Assumption A hold for a system of risky objects $V_{j}, j \in \underline{d}$, and let agent $i \in \underline{n}$ hold a portfolio with weights according to (5.1). Then for the Conditional Value-at-Risks with fixed thresholds $u, s \in(0, \infty)$ or with scaling factor $\theta \in\left(0, a_{i}\right)$ it holds asymptotically as level $\alpha \uparrow 1$ :

(i) for fixed thresholds $u, s \in(0, \infty)$ :

$$
\begin{aligned}
& \operatorname{Ind} \operatorname{CoVaR}_{U_{i} \mid S>s}(\alpha) \sim \frac{-a_{i} \ln (1-\alpha)}{\lambda_{i(1)}} \sim \operatorname{Ind} \operatorname{VaR}(\alpha), \\
& \operatorname{Sys}_{\operatorname{CoVaR}_{S \mid U_{i}>u}(\alpha)} \sim \frac{-\ln (1-\alpha)}{\lambda_{1}} \sim \operatorname{Sys} \operatorname{VaR}(\alpha) ;
\end{aligned}
$$

(ii) for proportionally increasing thresholds with scaling factor $\theta \in\left(0, a_{i}\right)$ :

$$
\begin{aligned}
&{\operatorname{Ind~} \operatorname{CoVaR}_{U_{i} \mid S}(\alpha, \theta)} \sim \frac{-a_{i} \ln (1-\alpha)}{\theta\left(\lambda_{i(1)}-\lambda_{1}\right)} \quad \text { for } \lambda_{1}<\lambda_{i(1)}, \\
& \operatorname{Sys~CoVaR}_{S \mid U_{i}}(\alpha, \theta) \sim \frac{-a_{i} \ln (1-\alpha)}{\left(a_{i}-\theta\right) \lambda_{1}} .
\end{aligned}
$$

We show in Theorem 5.3(i) that for fixed thresholds in the conditional events $\{S>$ $s\}$ or $\left\{U_{i}>u\right\}$ the asymptotic CoVaRs are independent of those thresholds $s, u$ and behave equivalent to the respective unconditional VaRs. To measure the asymptotic dependence between individual and system risk we provide in Theorem 5.3(ii) the modification of CoVaR based on conditional events with proportional increasing thresholds with scaling factor $\theta$. Here the system's influence on the individual agent's risk is determined by his portfolio weight $a_{i}$ and by the difference between the risk-dominant tail parameters $\lambda_{i(1)}$ of the agent's portfolio and $\lambda_{1}$ of the entire system. Note that if agent $i$ holds the dominant object with parameter $\lambda_{1}$, then the conditional distribution $P\left(U_{i}>\theta s \mid S>s\right)$ is degenerated as $P\left(U_{i}>\theta s, S>s\right) \sim$ $P(S>s)$ for $\theta \in\left(0, a_{i}\right), s \rightarrow \infty$ and, hence, a result for Ind $\operatorname{CoVaR}_{U_{i} \mid S}(\alpha, \theta)$ for $\lambda_{1}=\lambda_{i(1)}$ does not exist. In contrast, the system's CoVaR given an agent in trouble is asymptotically proportional to the system's VaR, whereby it depends on both portfolio weight $a_{i}$ and difference $a_{i}-\theta$. 
Remark 5.4. Note that our definition of CoVaR is different from those of Adrian and Brunnermeier [1] where the loss under condition is supposed to hit exactly some (high) value; i.e., the conditional events are of the form $\{S=s\}$ or $\left\{U_{i}=u\right\}$. Following the papers of Girardi and Ergün [17] and Mainik and Schaanning [29] we analyze CoVaR by conditioning on stress scenarios $\{S>s\}$ or $\left\{U_{i}>u\right\}$, which is more useful for our purposes as we discuss next.

In our system of independent $\mathcal{A E}$ losses the probability of the system loss $S>s$, given the exact loss value $u>0$ of an agent's portfolio is given by:

$$
P\left(S>s \mid U_{i}=u\right)=P\left(\sum_{k \in \underline{d} \backslash D_{i}} V_{k}>s-\frac{u}{a_{i}}\right) \sim \overline{C_{i}}\left(\frac{u}{a_{i}}\right) \exp \left(-\lambda_{\bar{i}(1)} s\right), s \rightarrow \infty,
$$

with $\overline{C_{i}}\left(u / a_{i}\right):=K_{V_{\bar{i}(1)}} \prod_{k \in \underline{d} \backslash D_{i}, k \neq \bar{i}(1)} \phi_{V_{k}}\left(\lambda_{\bar{i}(1)}\right) \exp \left(\lambda_{\bar{i}(1)} u / a_{i}\right)$, where $\bar{i}(1)$ is the index of the minimum tail parameter for objects in $\underline{d} \backslash D_{i}$. This shows that only the objects not held by agent $i$ influence the asymptotic conditional probability of the system loss. Hence, asymptotic results when conditioning on $\left\{U_{i}=u\right\}$ neglect potential severe object losses within the agent's portfolio. Moreover, this leads to $P\left(S>s \mid U_{i}=u\right)=o(P(S>s)), s \rightarrow \infty$, when $\lambda_{1}=\lambda_{i(1)}$, i.e. $\lambda_{1}<\lambda_{\bar{i}(1)}$. These problems can be avoided by using CoVaRs based on stress scenarios $\left\{U_{i}>u\right\}$ which lead due to result (5.3) for $s \rightarrow \infty$ to:

$$
P\left(S>s \mid U_{i}>u\right) \sim C_{i}\left(\frac{u}{a_{i}}\right) \exp \left(-\lambda_{1} s\right) / \sum_{j \in D_{i}} \pi_{j, d_{i}}\left(\frac{u}{a_{i}}\right) \exp \left(-\lambda_{j}\left(\frac{u}{a_{i}}\right)\right) .
$$

Now we provide our results on the Conditional Expected Shortfalls in the network context, namely the individual Conditional Expected Shortfall of agent $i \in \underline{n}$ :

$$
\text { Ind } \operatorname{CES}_{U_{i} \mid S}(\alpha):=E\left[U_{i} \mid S>\operatorname{Sys} \operatorname{VaR}(\alpha)\right],
$$

and the system Conditional Expected Shortfall:

$$
\operatorname{Sys}_{\operatorname{CES}} \mathrm{U}_{i}(\alpha):=E\left[S \mid U_{i}>\operatorname{Ind} \operatorname{VaR}_{i}(\alpha)\right] .
$$

These are the two most practically important CES measures: Ind $\mathrm{CES}_{U_{i} \mid S}$ is the expected loss of agent $i$ given that the financial system is in distress, and, hence, it can be used for comparing individual risks in a systemic crisis situation. Accordingly, Sys $\mathrm{CES}_{S \mid U_{i}}$ is the expected loss of the financial system given that the agent $i$ faces a high loss, and, hence, it is of a regulator's system stability interest. Note that CES statements on $E\left[U_{k} \mid U_{i}>\operatorname{Ind} \operatorname{VaR}_{i}(\alpha)\right]$ for two distinct agents $k$ and $i$ are of less practical relevance, as the (possibly competing) agents usually do not know the portfolio compositions of each other. Hence, we concentrate here on the CES results for $S$ and $U_{i}$. 
Theorem 5.5. Let Assumption A hold for a system of risky objects $V_{j}, j \in \underline{d}$, and let agent $i \in \underline{n}$ hold a portfolio with weights according to (5.1). Then it follows for the Conditional Expected Shortfalls as level $\alpha \uparrow 1$ :

(i) in case $\lambda_{1}=\lambda_{i(1)}$, where the risk dominant object is in the agent's portfolio:

$$
\text { Ind } \mathrm{CES}_{U_{i} \mid S}(\alpha) \sim-a_{i} \ln (1-\alpha) / \lambda_{1} \sim a_{i} \operatorname{Sys} \operatorname{ES}(\alpha),
$$

otherwise, for $\lambda_{1}<\lambda_{i(1)}$, it holds that:

$$
\begin{gathered}
\lim _{\alpha \uparrow 1} \operatorname{Ind} \operatorname{CES}_{U_{i} \mid S}(\alpha)=a_{i} \frac{\pi_{\bar{i}(1),\left(d-d_{i}\right)}}{\pi_{1, d}} \sum_{j \in D_{i}} \pi_{j, d_{i}} \frac{\lambda_{j}}{\left(\lambda_{j}-\lambda_{1}\right)^{2}}, \\
\text { with } \quad \pi_{\bar{i}(1),\left(d-d_{i}\right)}=K_{V_{\bar{i}(1)}} \prod_{k \in \underline{d} \backslash D_{i}, k \neq i(1)} \phi_{V_{k}}\left(\lambda_{\bar{i}(1)}\right), \quad \lambda_{\bar{i}(1)}=\min _{k \in \underline{d} \backslash D_{i}} \lambda_{k}, \text { and } \\
\pi_{j, d_{i}}=K_{V_{j}} \prod_{k \in D_{i}, k>j} \phi_{V_{k}}\left(\lambda_{j}\right) ;
\end{gathered}
$$

(ii) for the system CES, it holds that:

$$
\operatorname{Sys}_{\operatorname{CES}_{S \mid U_{i}}}(\alpha) \sim \frac{-\ln (1-\alpha)}{\lambda_{i(1)}} \sim \frac{1}{a_{i}} \operatorname{Ind} \operatorname{ES}_{i}(\alpha) .
$$

In Theorem 5.5(i) we point out an interesting phenomenon: the individual CES of agent $i$ depends qualitatively on whether he holds a proportion of the risk-dominant object in his portfolio or not. In the first case his CES is asymptotically proportional to the system's ES, in the second case it converges to a positive, finite limit. This implies that in a systemic crisis the individual expected loss of an agent holding the risk-dominant object increases proportionally to the Expected Shortfall of the system, i.e. the individual risk is unbounded; whereas agents avoiding the risk-dominant (i.e., the most "toxic") object has a finite (bounded) risk during a systemic crisis.

Conversely, in Theorem 5.5(ii) we give a measure for the influence of the individual agent's risk on the system risk, reflecting the impact of this agent on the entire system stability. The Conditional Expected Shortfall of the system, given an agent is in distress, is asymptotically proportional to the individual agent's Expected Shortfall. It depends only on the dominant tail parameter of the agent and, remarkably, is not affected by the chosen portfolio weight $a_{i}$.

Remark 5.6. In Figure 5.1 we visualize quantile-based risk measures for a system of $\mathcal{A E}$ object losses to illustrate the impact of an agent's portfolio structure on the system risk. For a four object system with tail parameters $\lambda_{1}=1, \lambda_{2}=4 / 3$, $\lambda_{3}=5 / 3, \lambda_{4}=2$ the asymptotic $\operatorname{ObjES}_{j}(\alpha), j=1,2,3,4$ are displayed in thin, dotted lines, and the asymptotic $\operatorname{Sys} \operatorname{ES}(\alpha)$ - which coincides with the asymptotic $\operatorname{Obj~ES}_{1}(\alpha)$ - in a thick, red line. Moreover, in plot (A) we consider agent 1 holding 

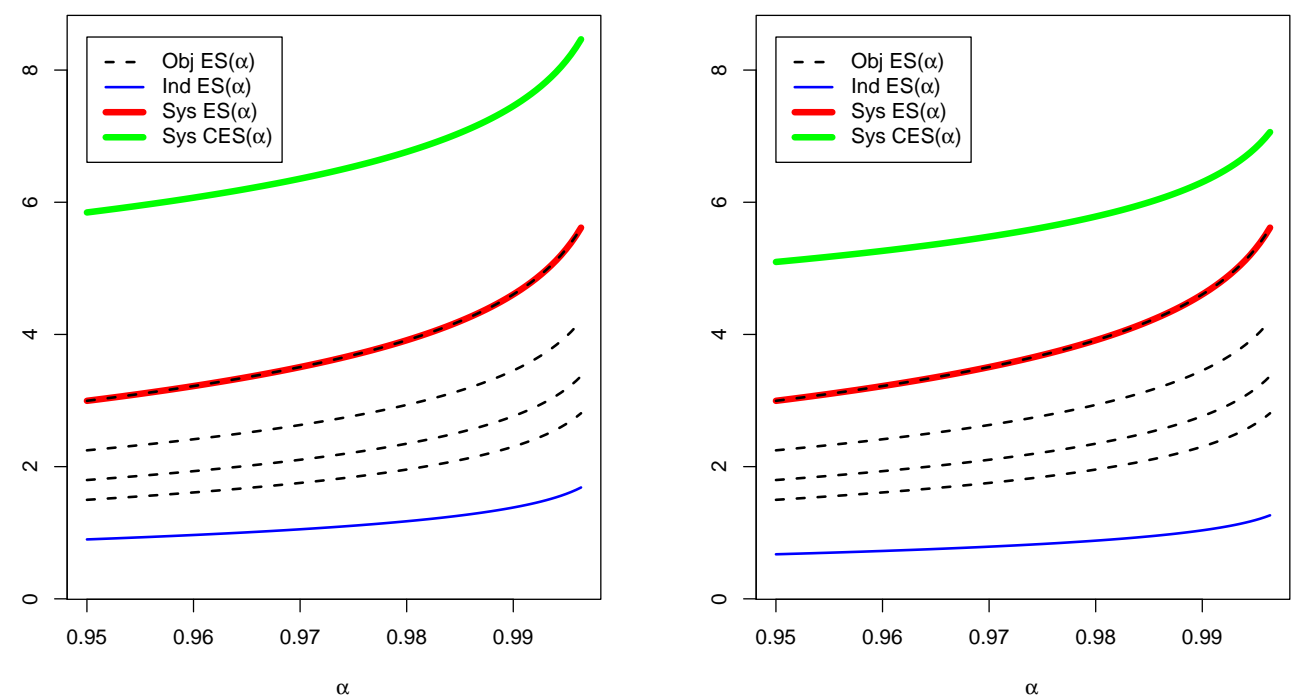

Figure 5.1: Asymptotic Expected Shortfalls for object, individual agent and system losses as well as system's Conditional Expected Shortfall given a large agent's loss for a portfolio with the riskiest object in (A) or the second-riskiest object in (B); for more details see Remark 5.6.

his dominant object 1 with $a_{1,1}=0.3$ and, hence, $\mu_{1}=\min _{j \in D_{1}}\left(\lambda_{1} / a_{1,1}\right)=0.3 \lambda_{1}=$ 0.3 , cf. (4.1); whereas in plot (B), we presume agent 2 to hold his dominant object 2 with $a_{2,2}=0.3$ and $\mu_{2}=0.3 \lambda_{2}=0.4$. Their asymptotic Expected Shortfalls are plotted in (A) and (B) in thin, blue lines. Note that, although agent 1 holds the riskiest object of the system and agent 2 only the second-riskiest one, their individual Expected Shortfall functions in (A) and (B) are rather similar. However, by plotting

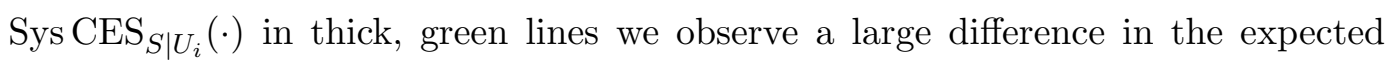
system loss depending on whether losses of agent 1 in (A) or of agent 2 in (B) are critical for the system.

\section{Comparison of results for light and heavy tails}

Finally, we contrast our results for a system of light-tailed $\mathcal{A E}$ risks with established results under the assumption of heavy-tailed risks. The settings in Kley et al. [23, 24] are suitable for such a comparison as they investigate risky systems of a similar structure. The essential difference from our setting is that the object losses are assumed there to be heavy-tailed asymptotically Pareto $(\mathcal{A P})$ distributed, with the same tail parameter $\gamma>0$ for all objects. Hence, we compare the results for systems 
with independent object losses $V_{j}, j \in \underline{d}$, for two settings satisfying for $x \rightarrow \infty$ :

$$
\begin{aligned}
& \text { Light tail } \mathcal{A E}\left(\lambda_{j}\right) \text {-assumption: } \quad P\left\{V_{j}>x\right\} \\
& \text { Heavy tail } \mathcal{A P}(\gamma) \text {-assumption: } \quad P\left\{V_{j}>x\right\}
\end{aligned}
$$

Our comparison is focussed on the three main issues: (I) tail parameters, (II) individual agent and system loss distributions, (III) risk measures.

(I) We underscore the different role of the tail parameters $\lambda_{j}$ and $\gamma$. They both describe the tail decay of the survival functions for object losses, however, act differently as scale and shape parameters, respectively. Scaling a loss with some weight $a>0$ changes the tail decay for $\mathcal{A E}$ losses but not for $\mathcal{A P}$ losses:

$$
\begin{aligned}
V \in \mathcal{A} \mathcal{E}(\lambda) & \Rightarrow a V \in \mathcal{A} \mathcal{E}(\lambda / a), \\
V \in \mathcal{A P}(\gamma) & \Rightarrow a V \in \mathcal{A P}(\gamma) .
\end{aligned}
$$

This difference is an essential one: in an $\mathcal{A} \mathcal{P}$-setting Kley et al. [23, 24] consider the same tail parameter $\gamma$ for all objects because losses with larger tail parameter are asymptotically negligible. This is not the case for an $\mathcal{A} \mathcal{E}$-setting: our Theorem 4.1 proves that object losses which are asymptotically negligible for the system can be dominant for agents' risk exposures, as scaling - holding only proportions of the risky objects in the portfolio - changes the tail decay.

(II) Next, we compare individual and system risks for both $\mathcal{A} \mathcal{E}$ - and $\mathcal{A P}$-settings. For $\mathcal{A E}$ object losses we prove that the individual agent's risk is determined asymptotically by the dominant object in his portfolio. The survival functions of the agents' risk exposures $U_{i}$ have generically distinct tail decays, which can differ from those of the system loss. This means that some individual risks are asymptotically of lower order compared to others and, in particular, to the system risk:

$$
\frac{P\left\{U_{i}>x\right\}}{P\{S>x\}} \rightarrow 0 \text { for } x \rightarrow \infty
$$

as we have shown in Theorem 4.1, see also (4.2), save for the special case that agent $i$ selects the most risky object $V_{1}$ alone with $a_{i, 1}=1$.

In contrast, for an $\mathcal{A P}$-setting individual and system risks are asymptotically proportional, see Theorem 3.2 in Kley et al. [23]:

$$
\frac{P\left\{U_{i}>x\right\}}{P\{S>x\}} \rightarrow \text { const }>0 \text { for } x \rightarrow \infty .
$$

Moreover, our analysis reveals another fundamental difference between the risk distribution in light tail $\mathcal{A} \mathcal{E}$ - or heavy tail $\mathcal{A P}$-settings. In Theorem 3.6 we prove that in an $\mathcal{A} \mathcal{E}$-setting, for both system and individual agent's losses, their asymptotic risk 
distributions depend not only on the asymptotic but also on the non-asymptotic behavior of the object loss distributions in terms of their moment-generating functions. Whereas in an $\mathcal{A} \mathcal{P}$-setting the evaluation of asymptotic risk distributions requires only the asymptotic distributions of object losses.

(III) Finally, we compare the results on marginal and conditional risk measures. For convenience, we focus on portfolios with equal proportions for all selected risky objects as defined in (5.1). In both $\mathcal{A} \mathcal{E}$ - and $\mathcal{A P}$ - settings, Value-at-Risk and Expected Shortfall for losses of objects, individual agents and of the entire system are asymptotically proportional to each other for each setting, respectively. They tend to infinity for level $\alpha \uparrow 1$ with logarithmic rates for $\mathcal{A} \mathcal{E}$-settings as we show in Proposition 4.3, and with power rates for $\mathcal{A P}$-settings (cf. Cor. 3.7 in Kley et al. [23]) as:

$$
\begin{aligned}
\operatorname{Obj}_{\operatorname{VaR}_{j}}(\alpha) & \sim K_{V_{j}}^{1 / \gamma}(1-\alpha)^{-1 / \gamma}, \\
\operatorname{Ind} \operatorname{VaR}_{i}(\alpha) & \sim a_{i}\left(\sum_{j \in D_{i}} K_{V_{j}}\right)^{1 / \gamma}(1-\alpha)^{-1 / \gamma}, \\
\operatorname{Sys} \operatorname{VaR}(\alpha) & \sim\left(\sum_{j \in \underline{d}} K_{V_{j}}\right)^{1 / \gamma}(1-\alpha)^{-1 / \gamma} .
\end{aligned}
$$

Moreover, in an $\mathcal{A P}$-setting the Value-at-Risk for objects, agents or the system is asymptotically proportional to the respective Expected Shortfall with the proportionality factor $\gamma /(\gamma-1)$ for $\gamma>1$, see Cor. 3.8 in Kley et al. [23]. In an $\mathcal{A} \mathcal{E}$-setting Value-at-Risk and Expected Shortfall are asymptotically equivalent, more precisely, Proposition 4.3(ii) proves that their difference converges to a finite, non-zero limit. Furthermore, in an $\mathcal{A} \mathcal{E}$-setting Value-at-Risks and Expected Shortfalls are independent of the factors $K_{V_{j}}$ in contrast to those in an $\mathcal{A P}$-setting.

Finally, we compare light tail $\mathcal{A} \mathcal{E}$ - and heavy tail $\mathcal{A} \mathcal{P}$-settings by analyzing the Conditional Expected Shortfalls within a financial network. For an $\mathcal{A P}$-setting, Kley et al. [24] show for heavy-tailed risks with finite mean, characterized by $\gamma>1$, that Sys $\operatorname{CES}_{S \mid U_{i}}(\alpha)=E\left[S \mid U_{i}>\operatorname{Ind} \operatorname{VaR}_{i}(\alpha)\right]$ is for $\alpha \uparrow 1$ asymptotically proportional to the unconditional individual Expected Shortfall $\operatorname{Ind} \operatorname{ES}_{i}(\alpha)$. This is also valid for an $\mathcal{A} \mathcal{E}$-setting as we derive in Theorem 5.5(ii). The situation is very different if we consider the impact of a systemic crisis on the individual agent's risk: In heavy-tail $\mathcal{A P}$-settings the agent's Ind $\mathrm{CES}_{U_{i} \mid S}(\alpha)=E\left[U_{i} \mid S>\operatorname{Sys} \operatorname{VaR}(\alpha)\right]$ always tends for $\alpha \uparrow 1$ to infinity as:

$$
\text { Ind } \mathrm{CES}_{U_{i} \mid S}(\alpha) \sim a_{i} \frac{\gamma}{\gamma-1}\left(\sum_{j \in D_{i}} K_{V_{j}}\right)\left(\sum_{j \in \underline{d}} K_{V_{j}}\right)^{1 / \gamma-1}(1-\alpha)^{-1 / \gamma} .
$$

This contrasts with our result in Theorem 5.5(i) which states for an $\mathcal{A} \mathcal{E}$-setting 
that Ind $\operatorname{CES}_{U_{i} \mid S}(\alpha)$ depends on the agent's portfolio composition. In particular, it increases proportionally to the Expected Shortfall of the system if the agent holds the risk-dominant object in his portfolio, but for all portfolios without the risk-dominant object it converges to a finite limit.

Hence, we have established substantial differences in heavy tail and light tail scenarios, which have to be accounted for in risk management and regulatory decisions.

\section{Proofs}

Proof of Lemma 3.5. Since the object loss $V_{k}, k \in \underline{d}$ are positive random variables, its moment-generating function $\phi_{V_{k}}(\cdot)$ exists at least for all $t \leq 0$. For $t \in\left(0, \lambda_{k}\right)$ we calculate

$$
\begin{aligned}
\phi_{V_{k}}(t) & =E\left[\exp \left(t V_{k}\right)\right]=1+\int_{0}^{\infty} P\left\{\exp \left(t V_{k}\right)-1>x\right\} \mathrm{d} x \\
& =1+\int_{1}^{\infty} K_{V_{k}}(\ln y / t) y^{-\lambda_{k} / t} \mathrm{~d} y=1+K_{V_{k}}(\xi) \frac{t}{\lambda_{k}-t}
\end{aligned}
$$

for some $\xi \in(0, \infty)$ by the mean value theorem. Since the $K_{V_{k}}(\cdot), k \in \underline{d}$ are continuous and strictly positive with $K_{V_{k}}(0)=1$ and $\lim _{x \rightarrow \infty} K_{V_{k}}(x)=K_{V_{k}} \in(0, \infty)$, it follows that the moment-generating function $\phi_{V_{k}}(\cdot)$ is finite for all $t<\lambda_{k}$ with a singularity at $\lambda_{k}$.

Proof of Theorems 3.2, 3.6, and Corollary 3.4. The results are proven by induction. We use that the convolution of $k \geq 2$ object losses can be calculated from the convolution of $(k-1)$ object losses recursively for $x>0$ as follows:

$$
P\left\{\sum_{j \in \underline{k}} V_{j}>x\right\}=P\left\{V_{k}>x\right\}+\int_{0}^{x} P\left\{\sum_{j \in \underline{k-1}} V_{j}>x-y\right\} \mathrm{d} F_{V_{k}}(y) .
$$

For $d=1$ object we obtain $P\{S>x\}=P\left\{V_{1}>x\right\}=\pi_{1,1}(x) \exp \left(-\lambda_{1} x\right)$, where $\pi_{1,1}(x):=K_{V_{1}}(x), x>0$, is bounded away from zero and infinity, cf. definition of $K_{V_{1}}(\cdot)$ in Eq. (2.5) and proof of Lemma 3.5. It holds $\lim _{x \rightarrow \infty} \pi_{1,1}(x)=K_{V_{1}} \in(0, \infty)$ due to Assumption A.

Now assume that the results of Theorems 3.2, 3.6 on $S$ are valid for $d=k-1$ objects for some $k \geq 2$ : it holds that $P\left\{\sum_{j \in k-1} V_{j}>x\right\}=\sum_{j \in k-1} \pi_{j, k-1}(x) \exp \left(-\lambda_{j} x\right)$ with positive functions $\pi_{j, k-1}(x), x>0$ of form (3.4), which are bounded from above and have limits $\pi_{j, k-1}:=\lim _{x \rightarrow \infty} \pi_{j, k-1}(x)=K_{V_{j}} \prod_{l=j+1}^{k-1} \phi_{V_{l}}\left(\lambda_{j}\right) \in(0, \infty)$ for all 
$j \in \underline{k-1}$, where $\phi_{V_{l}}(\cdot)$ denotes the moment-generating function of $V_{l}$. Then Eq. (7.1) implies for the convolution of $k$ object losses:

$$
\begin{aligned}
& P\left\{\sum_{j \in \underline{k}} V_{j}>x\right\} \\
& =K_{V_{k}}(x) \exp \left(-\lambda_{k} x\right)+\sum_{j \in \underline{k-1}} \int_{0}^{x} \pi_{j, k-1}(x-y) \exp \left(-\lambda_{j}(x-y)\right) \mathrm{d} F_{V_{k}}(y) \\
& =\sum_{j \in \underline{k}} \pi_{j, k}(x) \exp \left(-\lambda_{j} x\right),
\end{aligned}
$$

with functions

$$
\begin{aligned}
\pi_{j, k}(x) & :=\int_{0}^{x} \pi_{j, k-1}(x-y) \exp \left(\lambda_{j} y\right) \mathrm{d} F_{V_{k}}(y), \quad j \in \underline{k-1}, \\
\pi_{k, k}(x) & :=K_{V_{k}}(x) .
\end{aligned}
$$

The mean value theorem implies that there exist values $\xi_{j}(x) \in(0, x), j \in \underline{k-1}$, such that:

$$
\pi_{j, k}(x)=\pi_{j, k-1}\left(x-\xi_{j}(x)\right) \int_{0}^{x} \exp \left(\lambda_{j} y\right) \mathrm{d} F_{V_{k}}(y)=: \pi_{j, k-1}\left(x-\xi_{j}(x)\right) I_{j, k}(x) .
$$

Let $x_{0}$ be an arbitrary fixed value with $0<x_{0}<x$, then it holds for all $j \in \underline{k-1}$ that:

$$
\lim _{x \rightarrow \infty} \int_{x-x_{0}}^{x} \pi_{j, k-1}(x-y) \exp \left(\lambda_{j} y\right) \mathrm{d} F_{V_{k}}(y)=0,
$$

which follows from $1-F_{V_{k}}(x) \sim K_{V_{k}} \exp \left(-\lambda_{k} x\right), x \rightarrow \infty$, where $\lambda_{k}>\lambda_{j}$ for all $j \in \underline{k-1}$ (cf. Assumption A) and from the boundedness of $\pi_{j, k-1}(\cdot)$ as above.

The functions $I_{j, k}(x), j \in \underline{k-1}$, from (7.4) are strictly positive for $x>0$ because of the positive integrands, and converge for $x \rightarrow \infty$ to the finite values $\phi_{V_{k}}\left(\lambda_{j}\right)$ of the moment-generating function of $V_{k}$, where the finiteness is proven in Lemma 3.5. Hence, the $I_{j, k}(\cdot)$ and (consequently by Eq. (7.4)) the $\pi_{j, k}(\cdot), j \in \underline{k-1}$, are bounded from above. With (7.5) it follows for $j \in \underline{k-1}$ and $x \rightarrow \infty$ :

$$
\begin{aligned}
\pi_{j, k}(x) & \sim \int_{0}^{x-x_{0}} \pi_{j, k-1}(x-y) \exp \left(\lambda_{j} y\right) \mathrm{d} F_{V_{k}}(y) \\
& =\pi_{j, k-1}\left(x-\xi_{j}\left(x-x_{0}\right)\right) \int_{0}^{x-x_{0}} \exp \left(\lambda_{j} y\right) \mathrm{d} F_{V_{k}}(y),
\end{aligned}
$$

for arbitrary $0<x_{0}<x$ and mean values $\xi_{j}\left(x-x_{0}\right) \in\left(0, x-x_{0}\right)$. In the asymptotic analysis for $x \rightarrow \infty$ we can choose the value of $x_{0}$ and, hence, the value $x-\xi_{j}(x-$ 
$\left.x_{0}\right) \in\left(x_{0}, x\right)$ arbitrarily large. Together with $\lim _{x \rightarrow \infty} \pi_{j, k-1}(x)=\pi_{j, k-1} \in(0, \infty)$, we obtain for $j \in \underline{k-1}$ and $x \rightarrow \infty$ :

$$
\pi_{j, k}(x) \sim \pi_{j, k-1} \int_{0}^{x} \exp \left(\lambda_{j} y\right) \mathrm{d} F_{V_{k}}(y) \sim \pi_{j, k-1} \phi_{V_{k}}\left(\lambda_{j}\right) .
$$

Consequently, the mixing proportion functions $\pi_{j, k}(\cdot), j \in \underline{k}$, from Eqs. $(7.2)-(7.3)$ converge:

$$
\lim _{x \rightarrow \infty} \pi_{j, k}(x)=\pi_{j, k-1} \phi_{V_{k}}\left(\lambda_{j}\right)=K_{V_{j}} \prod_{l=j+1}^{k} \phi_{V_{l}}\left(\lambda_{j}\right)=: \pi_{j, k} \in(0, \infty),
$$

where we apply $\pi_{j, k-1}=K_{V_{j}} \prod_{l=j+1}^{k-1} \phi_{V_{l}}\left(\lambda_{j}\right), j \in \underline{k-1}$, as given above. For $j=k$ the empty product in (7.7) is set equal to 1 according to the common convention. Hence, the statements for convolution of $d=k$ object losses are deduced from those for $d=k-1$ objects.

Altogether, the results in Theorems 3.2 and 3.6 on $S$ are proven, and the results in Corollary 3.4 and Theorem 3.6 on $U_{i}$ follow analogously.

Theorem 4.1 follows from Theorem 3.6.

Proof of Proposition 4.3. Theorem 4.1 gives for $i \in \underline{n}$ and $x \rightarrow \infty$ :

$$
P\left\{U_{i}>x\right\} \sim K_{U_{i}} \exp \left(-\mu_{i} x\right)=: P_{i}(x),
$$

with constant $K_{U_{i}}>0$ given in statement (ii) of Theorem 4.1 and $\mu_{i}$ from (4.1). Hence, for the inverse it follows that:

$$
P_{i}^{-1}(y)=\frac{\ln \left(K_{U_{i}}\right)-\ln (y)}{\mu_{i}} \sim \frac{-\ln (y)}{\mu_{i}}, \quad y \downarrow 0 .
$$

Inserting $y=1-\alpha$ gives the asymptotic result for $\operatorname{Ind} \operatorname{VaR}_{i}(\alpha)$. The Value-at-Risks for object and system losses can be obtained analogously. Hence, the results in statement (i) of Proposition 4.3 are proven.

To deduce the results in statement (ii), we use the functional mixture representation provided in Theorem 3.6, cf. also Eq. (3.8), and obtain for $i \in \underline{n}$ and $u \rightarrow \infty$ :

$$
\begin{aligned}
& \int_{u}^{\infty} P\left\{U_{i}>x\right\} \mathrm{d} x=\sum_{j \in D_{i}} \int_{u}^{\infty} \pi_{i, j, d_{i}}(x) \exp \left(-\lambda_{j} x / a_{i, j}\right) \mathrm{d} x \\
& \sim \sum_{j \in D_{i}} \pi_{i, j, d_{i}} \int_{u}^{\infty} \exp \left(-\lambda_{j} x / a_{i, j}\right) \mathrm{d} x=\sum_{j \in D_{i}} \frac{a_{i, j} \pi_{i, j, d_{i}}}{\lambda_{j}} \exp \left(-\lambda_{j} u / a_{i, j}\right) \\
& \sim \frac{K_{U_{i}}}{\mu_{i}} \exp \left(-\mu_{i} u\right),
\end{aligned}
$$


with $\pi_{i, j, d_{i}}=\lim _{x \rightarrow \infty} \pi_{i, j, d_{i}}(x)$. The last step in (7.8) follows from Theorem 4.1(ii) with constant $K_{U_{i}}>0$ given there and $\mu_{i}$ from (4.1). Hence, we obtain for $u \rightarrow \infty$ :

$$
\begin{aligned}
E\left[U_{i} \mid U_{i}>u\right]-u & =\frac{E\left[\left(U_{i}-u\right) \mathbf{1}_{\left\{U_{i}-u>0\right\}}\right]}{P\left\{U_{i}>u\right\}}=\int_{u}^{\infty} \frac{P\left\{U_{i}>x\right\}}{P\left\{U_{i}>u\right\}} \mathrm{d} x \\
& \sim \frac{K_{U_{i}} / \mu_{i} \exp \left(-\mu_{i} u\right)}{K_{U_{i}} \exp \left(-\mu_{i} u\right)}=\frac{1}{\mu_{i}}
\end{aligned}
$$

with indicator function $\mathbf{1}_{\{\cdot\}}$. The result in statement (ii) of Proposition 4.3 on Ind $\mathrm{ES}_{i}$ follows by inserting $u=\operatorname{Ind} \operatorname{VaR}_{\mathrm{i}}(\alpha)$; the other results in (ii) can be obtained as the following special cases: on $\mathrm{ObjES}_{j}$ with $D_{i}=\{j\}, a_{i, j}=1, \mu_{i}=\lambda_{j}$; on Sys ES with $D_{i}=\underline{d}, a_{i, j}=1$ for all $j \in \underline{d}, \mu_{i}=\lambda_{1}$.

Proof of Proposition 5.1. Consider some agent $i \in \underline{n}$ and partition the objects $\left\{V_{j}, j \in \underline{d}\right\}$ into the two subsets $M_{i}:=\left\{V_{j}, j \in D_{i}\right\}$ of the objects selected by agent $i$ and $\bar{M}_{i}:=\left\{V_{k}, k \in \underline{d} \backslash D_{i}\right\}$ of the not-selected objects. Accordingly, we define two random variables $W_{i}:=U_{i} / a_{i}=\sum_{j \in D_{i}} V_{j}$ and $\bar{W}_{i}:=S-W_{i}=\sum_{k \in \underline{d} \backslash D_{i}} V_{k}$ which are stochastically independent and follow $\mathcal{A E}$ distributions given for $x>0$ by (cf. $(3.3))$ :

$$
\begin{aligned}
P\left\{W_{i}>x\right\} & =\sum_{j \in D_{i}} \pi_{j, d_{i}}(x) \exp \left(-\lambda_{j} x\right) \\
P\left\{\bar{W}_{i}>x\right\} & =\sum_{k \in\left(\underline{d} \backslash D_{i}\right)} \pi_{k,\left(d-d_{i}\right)}(x) \exp \left(-\lambda_{k} x\right) .
\end{aligned}
$$

Here $\pi_{j, d_{i}}(x), j \in D_{i}$, are the mixing proportion functions corresponding to the subsystem formed by object set $M_{i}$, while $\pi_{k,\left(d-d_{i}\right)}(x), k \in \underline{d} \backslash D_{i}$, are those corresponding to the sub-system formed by object set $\bar{M}_{i}$.

For $0 \leq u \leq a_{i} s$ we obtain with (7.9) and (7.10) for some mean value $\bar{\xi}_{u / a_{i}}(s) \in$ $\left(u / a_{i}, s\right)$ that:

$$
\begin{aligned}
& P\left(U_{i}>u, S>s\right)=P\left(W_{i}>u / a_{i}, W_{i}+\bar{W}_{i}>s\right) \\
& =P\left(W_{i}>s\right)+\int_{u / a_{i}}^{s} P\left(\bar{W}_{i}>s-z\right) \mathrm{d} F_{W_{i}}(z)=\sum_{j \in D_{i}} \pi_{j, d_{i}}(s) \exp \left(-\lambda_{j} s\right) \\
& +\sum_{k \in \underline{d} \backslash D_{i}} \pi_{k,\left(d-d_{i}\right)}\left(s-\bar{\xi}_{u / a_{i}}(s)\right) \int_{u / a_{i}}^{s} \exp \left(-\lambda_{k}(s-z)\right) \mathrm{d} F_{W_{i}}(z) .
\end{aligned}
$$


For the integral in (7.11), by partial integration, (7.9), and again the mean value theorem we obtain for some $\xi_{u / a_{i}}(s) \in\left(u / a_{i}, s\right)$ :

$$
\begin{aligned}
& \int_{u / a_{i}}^{s} \exp \left(-\lambda_{k}(s-z)\right) \mathrm{d} F_{W_{i}}(z)=\left.\sum_{j \in D_{i}} \pi_{j, d_{i}}(z) \exp \left(-\lambda_{j} z\right) \exp \left(-\lambda_{k}(s-z)\right)\right|_{u / a_{i}} ^{s} \\
& -\sum_{j \in D_{i}} \int_{u / a_{i}}^{s} \pi_{j, d_{i}}(z) \exp \left(-\lambda_{j} z\right) \lambda_{k} \exp \left(-\lambda_{k}(s-z)\right) \mathrm{d} z \\
& =\sum_{j \in D_{i}} \exp \left(-\lambda_{j} s\right)\left(\pi_{j, d_{i}}(s)-\frac{\lambda_{k}}{\lambda_{k}-\lambda_{j}} \pi_{j, d_{i}}\left(\xi_{u / a_{i}}(s)\right)\right) \\
& \quad+\exp \left(-\lambda_{k} s\right) \sum_{j \in D_{i}}\left[\exp \left(\left(\lambda_{k}-\lambda_{j}\right) \frac{u}{a_{i}}\right)\left(\frac{\lambda_{k}}{\lambda_{k}-\lambda_{j}} \pi_{j, d_{i}}\left(\xi_{u / a_{i}}(s)\right)-\pi_{j, d_{i}}\left(\frac{u}{a_{i}}\right)\right)\right] .
\end{aligned}
$$

Consequently, we obtain with (7.11) that the joint distribution of $U_{i}$ and $S$ has a functional exponential mixture representation:

$$
P\left(U_{i}>u, S>s\right)=: \sum_{j \in \underline{d}} b_{j, u / a_{i}}(s) \exp \left(-\lambda_{j} s\right),
$$

where

$$
b_{j, u / a_{i}}(s)=\left\{\begin{aligned}
\pi_{j, d_{i}}(s) & +\sum_{k \in \underline{d} \backslash D_{i}} \pi_{k,\left(d-d_{i}\right)}\left(s-\bar{\xi}_{u / a_{i}}(s)\right) \\
\times\left(\pi_{j, d_{i}}(s)-\frac{\lambda_{k}}{\lambda_{k}-\lambda_{j}} \pi_{j, d_{i}}\left(\xi_{u / a_{i}}(s)\right)\right) & \text { for } j \in D_{i}, \\
\sum_{k \in D_{i}} \exp \left(\left(\lambda_{j}-\lambda_{k}\right) \frac{u}{a_{i}}\right) \pi_{j,\left(d-d_{i}\right)}\left(s-\bar{\xi}_{u / a_{i}}(s)\right) & \\
\times\left(\frac{\lambda_{j}}{\lambda_{j}-\lambda_{k}} \pi_{k, d_{i}}\left(\xi_{u / a_{i}}(s)\right)-\pi_{k, d_{i}}\left(\frac{u}{a_{i}}\right)\right) & \text { for } j \in \underline{d} \backslash D_{i},
\end{aligned}\right.
$$

where $b_{j, u / a_{i}}:[0, \infty) \rightarrow(-\infty, \infty), j \in \underline{d}$, are bounded functions.

Recall from Eq. (2.4) that the tail parameters are ordered such that $\lambda_{j}<\lambda_{k}$ for $j<k$. Hence, representation (7.12) implies that for the asymptotic analysis of $P\left(U_{i}>u, S>s\right)$ only the asymptotic behavior of the function $b_{1, u / a_{i}}(\cdot)$ in the dominant term $\exp \left(-\lambda_{1} s\right)$ matters and depends on whether the most risky object is in the agent $i$ portfolio or not. Denote by $\lambda_{i(1)}=\min _{j \in D_{i}} \lambda_{j}$, then we distinguish two cases:

$$
\text { Case I. } \lambda_{1}=\lambda_{i(1)}: 1 \in D_{i}, \quad \text { Case II. } \lambda_{1}<\lambda_{i(1)}: \quad 1 \in \underline{d} \backslash D_{i},
$$

We start with Case II, where $\lambda_{1}<\lambda_{j}$ for all $j \in D_{i}$ and obtain the finite limit:

$$
\lim _{s \rightarrow \infty} \int_{u / a_{i}}^{s} \exp \left(\lambda_{1} z\right) \mathrm{d} F_{W_{i}}(z)=\prod_{j \in D_{i}} \phi_{V_{j}}\left(\lambda_{1}\right)-\int_{0}^{u / a_{i}} \exp \left(\lambda_{1} z\right) \mathrm{d} F_{W_{i}}(z) \in(0, \infty),
$$


with moment-generating function $\phi_{W_{i}}(\cdot)=\prod_{j \in D_{i}} \phi_{V_{j}}(\cdot)$. Now we go back to (7.11), noticing that the dominant term is the summand for $k=1$ in the second term. The same argument as in (7.6) applies giving

$$
\lim _{s \rightarrow \infty} \pi_{1,\left(d-d_{i}\right)}\left(s-\bar{\xi}_{u / a_{i}}(s)\right)=\pi_{1,\left(d-d_{i}\right)} \in(0, \infty)
$$

Consequently, with (7.14) for $s \rightarrow \infty$ :

$$
\begin{aligned}
& P\left(U_{i}>u, S>s\right) \sim \pi_{1,\left(d-d_{i}\right)} \exp \left(-\lambda_{1} s\right) \int_{u / a_{i}}^{s} \exp \left(\lambda_{1} z\right) \mathrm{d} F_{W_{i}}(z) \\
& \sim \pi_{1,\left(d-d_{i}\right)} \exp \left(-\lambda_{1} s\right)\left(\prod_{j \in D_{i}} \phi_{V_{j}}\left(\lambda_{1}\right)-\int_{0}^{u / a_{i}} \exp \left(\lambda_{1} z\right) \mathrm{d} F_{W_{i}}(z)\right) \\
& \sim \exp \left(-\lambda_{1} s\right)\left(\pi_{1, d}-K_{V_{1}} \prod_{k \in \underline{d} \backslash D_{i}, k \neq 1} \phi_{V_{k}}\left(\lambda_{1}\right) \int_{0}^{u / a_{i}} \exp \left(\lambda_{1} z\right) \mathrm{d} F_{W_{i}}(z)\right),
\end{aligned}
$$

where in the last step we have adapted (3.10) to the situation, which gives $\pi_{1,\left(d-d_{i}\right)}=$ $K_{V_{1}} \prod_{k \in \underline{d} \backslash D_{i}, k \neq 1} \phi_{V_{k}}\left(\lambda_{1}\right)$.

For Case I we start with the following partition:

$$
\begin{aligned}
& P\left(U_{i}>u, S>s\right)=P\left(W_{i}>u / a_{i}, W_{i}+\bar{W}_{i}>s\right) \\
= & \int_{0}^{\infty} P\left(W_{i}>\max \left(u / a_{i}, s-z\right)\right) \mathrm{d} F_{\bar{W}_{i}}(z) \\
= & \int_{0}^{s-u / a_{i}} P\left(W_{i}>s-z\right) \mathrm{d} F_{\bar{W}_{i}}(z)+P\left(W_{i}>u / a_{i}\right) P\left(\bar{W}_{i}>s-u / a_{i}\right) \\
= & \sum_{j \in D_{i}} \exp \left(-\lambda_{j} s\right) \int_{0}^{s-u / a_{i}} \pi_{j, d_{i}}(s-z) \exp \left(\lambda_{j} z\right) \mathrm{d} F_{\bar{W}_{i}}(z) \\
& +\sum_{k \in \underline{d} \backslash D_{i}} \exp \left(-\lambda_{k} s\right) \sum_{j \in D_{i}} \pi_{j, d_{i}}\left(u / a_{i}\right) \pi_{k,\left(d-d_{i}\right)}\left(s-u / a_{i}\right) \exp \left(\left(\lambda_{k}-\lambda_{j}\right) u / a_{i}\right) .
\end{aligned}
$$

Since $1 \in D_{i}$ and $\lambda_{1}<\lambda_{k}$ for all $k \in \underline{d} \backslash D_{i}$, the moment-generating function $\phi_{\bar{W}_{i}}(\cdot)=\prod_{k \in \underline{d} \backslash D_{i}} \phi_{V_{k}}(\cdot)$ is finite at $\lambda_{1}$, such that for $s \rightarrow \infty$ :

$$
\begin{aligned}
& P\left(U_{i}>u, S>s\right) \sim \exp \left(-\lambda_{1} s\right) \int_{0}^{s-u / a_{i}} \pi_{1, d_{i}}(s-z) \exp \left(\lambda_{1} z\right) \mathrm{d} F_{\bar{W}_{i}}(z) \\
& \sim \exp \left(-\lambda_{1} s\right) \pi_{1, d_{i}} \prod_{k \in \underline{d} \backslash D_{i}} \phi_{V_{k}}\left(\lambda_{1}\right)=\pi_{1, d} \exp \left(-\lambda_{1} s\right)
\end{aligned}
$$


where the limit value $\pi_{1, d_{i}}$ follows as in (7.15).

Hereby, statement (i) for $u \leq a_{i} s$ and statement (ii) in Proposition 5.1 are proven. Statement (i) for $u>a_{i} s$ and statement (iii) follows, since for $u>a_{i} s$ :

$$
\begin{aligned}
P\left(U_{i}>u, S>s\right) & =P\left(W_{i}>u / a_{i}\right)=\sum_{j \in D_{i}} \pi_{j, d_{i}}\left(u / a_{i}\right) \exp \left(-\lambda_{j} u / a_{i}\right) \\
& \sim \pi_{i(1), d_{i}} \exp \left(-\lambda_{i(1)} u / a_{i}\right), \quad \text { for } u \rightarrow \infty .
\end{aligned}
$$

Proof of Theorem 5.3. From the results on the joint distribution of $U_{i}$ and $S$ as given in Proposition 5.1 it follows for the conditional probabilities that:

$$
\begin{aligned}
P\left(U_{i}>u \mid S>s\right) & \sim \pi_{i(1), d_{i}} /\left(\sum_{j \in \underline{d}} \pi_{j, d}(s) \exp \left(-\lambda_{j} s\right)\right) \exp \left(-\lambda_{i(1)} u / a_{i}\right), u \rightarrow \infty \\
P\left(S>s \mid U_{i}>u\right) & \sim C_{i}\left(\frac{u}{a_{i}}\right) /\left(\sum_{j \in D_{i}} \pi_{j, d}\left(\frac{u}{a_{i}}\right) \exp \left(-\frac{\lambda_{j} u}{a_{i}}\right)\right) \exp \left(-\lambda_{1} s\right), s \rightarrow \infty
\end{aligned}
$$

Then the CoVaR results of statement (i) in Theorem 5.3 follow analogously to the VaR results in Proposition 4.3.

To prove the results of statement (ii) we analyze the functional exponential mixture representation (which follows from Eq. (7.12) in the proof of Proposition 5.1) such that for $s>0$ and $\theta \in\left(0, a_{i}\right)$ :

$$
\begin{aligned}
P\left(U_{i}\right. & >\theta s, S>s)=\sum_{j \in D_{i}} b_{j, \theta s / a_{i}}(s) \exp \left(-\lambda_{j} s\right) \\
& +\sum_{j \in D_{i}} \sum_{k \in \underline{d} \backslash D_{i}} B_{j, k}\left(s, \theta s / a_{i}\right) \exp \left(-\left(\lambda_{j} \theta / a_{i}+\lambda_{k}\left(1-\theta / a_{i}\right)\right) s\right),
\end{aligned}
$$

with $B_{j, k}\left(s, u / a_{i}\right):=b_{k, u / a_{i}}(s) \exp \left(-\left(\lambda_{k}-\lambda_{j}\right) u / a_{i}\right)$ which is a bounded function in both arguments $s$ and $u / a_{i}$. Comparison of the exponents in (7.18) yields that $\lambda_{j}<\lambda_{j} \theta / a_{i}+\lambda_{k}\left(1-\theta / a_{i}\right)$ is equivalent to $\lambda_{j}<\lambda_{k}$, which implies:

$P\left(U_{i}>\theta s, S>s\right) \sim \begin{cases}\pi_{1, d} \exp \left(-\lambda_{1} s\right) & \text { for } \lambda_{1}=\lambda_{i(1)} \\ B_{i(1), 1} \exp \left(-\left(\lambda_{j} \theta / a_{i}+\lambda_{1}\left(1-\theta / a_{i}\right)\right) s\right) & \text { for } \lambda_{1}<\lambda_{i(1)}\end{cases}$

Here we apply $\lim _{s \rightarrow \infty} b_{1, \theta s / a_{i}}(s)=\pi_{1, d}$ and $\lim _{s \rightarrow \infty} B_{i(1), 1}\left(s, \theta s / a_{i}\right)=B_{i(1), 1} \in(0, \infty)$. The quantile functions of $P\left(U_{i}>\theta s \mid S>s\right)$ and $P\left(S>s \mid U_{i}>\theta s\right)$ - which could be obtained from (7.19) - yield the CoVaR results in Theorem 5.3(ii). 
Proof of Theorem 5.5. We apply representation (7.18), see proof of Theorem 5.3 above, for $\theta s=u>0$, and obtain that:

$$
\begin{gathered}
P\left(U_{i}>u\right) E\left[S \mid U_{i}>u\right]=\left(u / a_{i}\right) P\left(W_{i}>u / a_{i}\right)+\int_{u / a_{i}}^{\infty} P\left(U_{i}>u, S>s\right) \mathrm{d} s \\
=u / a_{i} \sum_{j \in D_{i}} \pi_{j, d_{i}}\left(u / a_{i}\right) \exp \left(-\lambda_{j} u / a_{i}\right)+\sum_{j \in D_{i}} \int_{u / a_{i}}^{\infty}\left(b_{j, u / a_{i}}(s) \exp \left(-\lambda_{j} s\right)\right. \\
\left.\quad+\sum_{k \in \underline{d} \backslash D_{i}} B_{j, k}\left(s, u / a_{i}\right) \exp \left(\left(\lambda_{k}-\lambda_{j}\right) u / a_{i}\right) \exp \left(-\lambda_{k} s\right)\right) \mathrm{d} s \\
\sim u / a_{i} \pi_{i(1), d_{i}} \exp \left(-\lambda_{i(1)} u / a_{i}\right), \quad \text { for } u \rightarrow \infty .
\end{gathered}
$$

By inserting $u=\operatorname{Ind} \operatorname{VaR}_{i}(\alpha) \sim \operatorname{IndES}_{i}(\alpha), \alpha \uparrow 1$ (see Corollary 4.5) this proves statement (ii) of Theorem 5.5.

The results in statement (i) can be deduced as follows: For $\lambda_{1}=\lambda_{i(1)}$ we obtain with the mean values $\xi(s) \in(0, s), \bar{\xi}(s) \in(s, \infty)$ that:

$$
\begin{aligned}
& P(S>s) E\left[U_{i} \mid S>s\right]=\int_{0}^{a_{i} s} P\left(U_{i}>u, S>s\right) \mathrm{d} u+\int_{a_{i} s}^{\infty} P\left(U_{i}>u\right) \mathrm{d} u \\
& =\sum_{j \in D_{i}} \exp \left(-\lambda_{j} s\right) b_{j, \xi(s)}(s) a_{i} s+\sum_{j \in D_{i}} a_{i} \exp \left(-\lambda_{j} s\right)\left(\frac{\pi_{j, d_{i}}(\bar{\xi}(s))}{\lambda_{j}}\right. \\
& \left.\quad+\sum_{k \in \underline{d} \backslash D_{i}} \frac{B_{j, k}(s, \xi(s))}{\lambda_{k}-\lambda_{j}}\right)-\sum_{k \in \underline{d} \backslash D_{i}} \exp \left(-\lambda_{k} s\right) \sum_{j \in D_{i}} \frac{a_{i} B_{j, k}(s, \xi(s))}{\lambda_{k}-\lambda_{j}} \\
& \sim a_{i} \pi_{1, d} s \exp \left(-\lambda_{1} s\right), \quad \text { for } s \rightarrow \infty,
\end{aligned}
$$

where we apply $\lim _{s \rightarrow \infty} b_{j, \xi(s)}(s)=\pi_{1, d}$ which has be shown in the proof of Proposition 5.1, see (7.16). This gives statement (i) for case $\lambda_{1}=\lambda_{i(1)}$.

To prove the corresponding result for case $\lambda_{1}<\lambda_{i(1)}$, we further exploit the property that $P\left(U_{i}>u, S>s\right) \sim \pi_{1,\left(d-d_{i}\right)} \exp \left(-\lambda_{1} s\right) \int_{u / a_{i}}^{s} \exp \left(\lambda_{1} z\right) \mathrm{d} F_{W_{i}}(z)$ holds in this case (see (7.15)), and obtain by changing the order of integrals that:

$$
\begin{aligned}
& \int_{0}^{a_{i} s} \pi_{1,\left(d-d_{i}\right)} \exp \left(-\lambda_{1} s\right) \int_{u / a_{i}}^{s} \exp \left(\lambda_{1} z\right) \mathrm{d} F_{W_{i}}(z) \mathrm{d} u \\
= & \pi_{1,\left(d-d_{i}\right)} \exp \left(-\lambda_{1} s\right) \int_{0}^{s} a_{i} z \exp \left(\lambda_{1} z\right) \mathrm{d} F_{W_{i}}(z) .
\end{aligned}
$$


Repeatedly applying integration by parts, we obtain for $s \rightarrow \infty$ :

$$
\begin{aligned}
& \int_{0}^{s} z \exp \left(\lambda_{1} z\right) \mathrm{d} F_{W_{i}}(z) \\
= & -\sum_{j \in D_{i}}\left[\pi_{j, d_{i}}(s) s \exp \left(-\left(\lambda_{j}-\lambda_{1}\right) s\right)+\int_{0}^{s} \pi_{j, d_{i}}(z)\left(1+\lambda_{1} z\right) \exp \left(-\left(\lambda_{j}-\lambda_{1}\right) z\right) \mathrm{d} z\right] \\
\sim & \sum_{j \in D_{i}} \pi_{j, d_{i}}\left[\exp \left(-\left(\lambda_{j}-\lambda_{1}\right) s\right)\left(-s-\frac{1+\lambda_{1} s}{\lambda_{j}-\lambda_{1}}-\frac{\lambda_{1}}{\left(\lambda_{j}-\lambda_{1}\right)^{2}}\right)+\frac{1}{\lambda_{j}-\lambda_{1}}+\frac{\lambda_{1}}{\left(\lambda_{j}-\lambda_{1}\right)^{2}}\right] \\
\rightarrow & \sum_{j \in D_{i}} \pi_{j, d_{i}} \frac{\lambda_{j}}{\left(\lambda_{j}-\lambda_{1}\right)^{2}} .
\end{aligned}
$$

Together with (7.20) and (7.21) we obtain for $s \rightarrow \infty$ that:

$$
P(S>s) E\left[U_{i} \mid S>s\right] \sim\left(a_{i} \pi_{1,\left(d-d_{i}\right)} \sum_{j \in D_{i}} \pi_{j, d_{i}} \frac{\lambda_{j}}{\left(\lambda_{j}-\lambda_{1}\right)^{2}}\right) \exp \left(-\lambda_{1} s\right),
$$

which gives the result for Ind $\mathrm{CES}_{U_{i} \mid S}(\alpha)$ in case $\lambda_{1}<\lambda_{i(1)}$. Therefore the results of Theorem 5.5 are proven.

Acknowledgments. This research has been in part financially supported by the Collaborative Research Center "Statistical modeling of nonlinear dynamic processes" (SFB 823, Teilprojekt A1) of the German Research Foundation (DFG).

\section{References}

[1] Adrian, T., Brunnermeier, M.K.: CoVaR. Am. Econ. Rev. 106(7), 1705-1741 (2016)

[2] Andersen, T.G., Davis, R.A., Kreiss, J.-P., Mikosch, T. (Eds.): Handbook of Financial Time Series. Springer, Berlin (2009)

[3] Asmussen, S., Albrecher, H.: Ruin Probabilities. World Scientific, New Jersey (2010)

[4] Barndorff-Nielsen, O.E., Mikosch, T., Resnick, S.I. (Eds.): Lévy Processes: Theory and Applications. Birkhäuser, Boston (2001)

[5] Behme, A., Klüppelberg, C., Reinert, G.: Hitting probabilities for compound Poisson processes in a bipartite network. ArXiv:1805.12459 (2018)

[6] Bergel, A.I., Egídio dos Reis, A.D.: Ruin problems in the generalized $\operatorname{Erlang}(n)$ risk model. Eur. Actuar. J. 6(1), 257-275 (2016)

[7] Brechmann, E.C., Hendrich, K., Czado, C.: Conditional copula simulation for systemic risk stress testing. Insur. Math. Econ. 53(3), 722-732 (2013)

[8] Coles, S., Heffernan, J., Tawn, J.: Dependence measures for extreme value analyses. Extremes 2(4), 339-365 (1999)

[9] Cont, R.: Empirical properties of asset returns: stylized facts and statistical issues. Quant. Financ. 1(2), 223-236 (2001) 
[10] Cont, R., Tankov, P.: Financial Modelling with Jump Processes. Chapman and Hall, CRC Press, Boca Raton (2004)

[11] Das, B., Fasen-Hartmann, V.: Risk contagion under regular variation and asymptotic tail independence. J. Multivariate Anal. 165, 194-215 (2018)

[12] Dȩbicki, K., Farkas, J., Hashorva, E.: Extremes of randomly scaled Gumbel risks. J. Math. Anal. Appl. 458(1), 30-42 (2018)

[13] Embrechts, P., Klüppelberg, C., Mikosch, T.: Modelling Extremal Events for Insurance and Finance. Springer, Berlin (1997)

[14] Embrechts, P., Wang, B., Wang, R.: Aggregation-robustness and model uncertainty of regulatory risk measures. Finance Stoch. 19(4), 763-790 (2015)

[15] Farkas, J., Hashorva, E: Tail approximation for reinsurance portfolios of Gaussian-like risks. Scand. Actuar. J. 2015(4), 319-331 (2015)

[16] Geluk, J.L., Peng, L., de Vries, C.G.: Convolutions of heavy-tailed random variables and applications to portfolio diversification and $\mathrm{MA}(1)$ time series. Adv. Appl. Prob. 32(4), 1011-1026 (2000)

[17] Girardi, G., Ergün, A.T.: Systemic risk measurement: Multivariate GARCH estimation of CoVaR. J. Bank. Financ. 37, 3169-3180 (2013)

[18] Hernández, C., Junca, M.: Optimal dividend payments under a time of ruin constraint: Exponential claims. Insur. Math. Econ. 65, 136-142 (2015)

[19] Ibragimov, R.: Portfolio diversification and value at risk under thick-tailedness. Quant. Financ. 9(5), 565-580 (2009)

[20] Jewell, N.P.: Mixtures of exponential distributions. Ann. Stat. 10, 479-484 (1982)

[21] Jiang, J., Tang, Q.: Reinsurance under the LCR and ECOMOR treaties with emphasis on light-tailed claims. Insur. Math. Econ. 43(3), 431-436 (2008)

[22] Kaas, R., Goovaerts, M., Dhaene, J., Denuit, M.: Modern Actuarial Risk Theory. Springer, 2nd ed., Berlin (2008)

[23] Kley, O., Klüppelberg, C., Reinert, G.: Risk in a large claims insurance market with bipartite graph structure. Oper. Res. 64(5), 1159-1176 (2016)

[24] Kley, O., Klüppelberg, C., Reinert, G.: Conditional risk measures in a bipartite market structure. Scand. Actuar. J. 2018(4), 328-355 (2018)

[25] Kyprianou, A.E.: Introductory Lectures on Fluctuations of Lévy Processes with Applications. Springer, Berlin (2006)

[26] Lin, E.M.H., Sun, E.W., Yu, M.: Systemic risk, financial markets, and performance of financial institutions. Ann. Oper. Res., 262(2), 579-603 (2018)

[27] Ly Vath, V., Mnif, M., Pham, H.: A model of optimal portfolio selection under liquidity risk and price impact. Finance Stoch. 11(1), 51-90 (2007)

[28] Mainik, G., Rüschendorf, L.: On optimal portfolio diversification with respect to extreme risks. Finance Stoch. 14(4), 593-623 (2010)

[29] Mainik, G., Schaanning, E.: On dependence consistency of CoVaR and some other systemic risk measures. Stat. Risk Model. 31(1), 49-77 (2014) 
[30] McLachlan, G.J.: Mixtures - models and applications. In: Balakrishnan, N. and Basu, A.P. (Eds.), The Exponential Distribution: Theory, Methods and Applications. Gordon and Breach, Amsterdam, 307-325 (1995)

[31] McNeil, A.J., Frey, R., Embrechts, P.: Quantitative Risk Management: Concepts, Techniques and Tools. Princeton University Press, New Jersey (2015)

[32] Mitra, A., Resnick, S.I.: Aggregation of rapidly varying risks and asymptotic independence. Adv. Appl. Probab. 41(3), 797-828 (2009)

[33] Xia, J.: Multi-agent investment in incomplete markets. Finance Stoch. 8(2), 241-259 (2004) 

